TRANSACTIONS OF THE

AMERICAN MATHEMATICAL SOCIETY

Volume 353, Number 3, Pages 1221-1249

S 0002-9947(00)02613-1

Article electronically published on November 16, 2000

\title{
REPRESENTATIONS AS ELEMENTS IN AFFINE COMPOSITION ALGEBRAS
}

\author{
PU ZHANG
}

\begin{abstract}
Let $A$ be the path algebra of a Euclidean quiver over a finite field $k$. The aim of this paper is to classify the modules $M$ with the property $[M] \in \mathcal{C}(A)$, where $\mathcal{C}(A)$ is Ringel's composition algebra. Namely, the main result says that if $|k| \neq 2,3$, then $[M] \in \mathcal{C}(A)$ if and only if the regular direct summand of $M$ is a direct sum of modules from non-homogeneous tubes with quasi-dimension vectors non-sincere. The main methods are representation theory of affine quivers, the structure of triangular decompositions of tame composition algebras, and the invariant subspaces of skew derivations. As an application, we see that $\mathcal{C}(A)=\mathcal{H}(A)$ if and only if the quiver of $A$ is of Dynkin type.
\end{abstract}

\section{INTRODUCTION}

0.1. The Ringel-Hall algebra approach to quantum groups is based on the following fact. Given any symmetrizable generalized Cartan matrix $\triangle$ in Kac's sense [K1, or equivalently, any symmetrizable Cartan datum in Lusztig's sense [L3], one can obtain a finite-dimensional hereditary algebra $A$ of type $\triangle$ over any finite field $k$, say, of $q$ elements. Then, by using all $A$-representations as a basis, one can construct the so-called Hall algebra $\mathcal{H}(A)$, and by varying $k$ and taking the cardinality $q$ as the quantum parameter, one can embed the positive part $U^{+}$of the DrinfeldJimbo algebra $U=U^{-} \otimes U^{0} \otimes U^{+}$of type $\triangle$ into $\mathcal{H}(A)$. This embedding sends the generators of $U^{+}$to the simple $A$-modules, and the corresponding image is exactly Ringel's composition algebra $\mathcal{C}(A)$. This is one of the main contributions of $\mathrm{C}$. M. Ringel and J. A. Green in R4, R5], R7- R10 and G1, G2]. This idea has led to several important advances in quantum groups, see e.g. [L1], [L2], L3].

This approach to quantum groups has the following two main advantages: first, it can be used without any modification not only for the symmetric GCMs, which correspond to the path algebras of quivers, but also for arbitrary symmetrizable GCMs, which correspond to the tensor algebras of species; second, if a representation $M$ can be an element in $U^{+}$, then the specific properties of $M$ as a representation can be carried to $U^{+}$-in particular, for example, one can apply the Auslander-Reiten theory, and on the other hand, a representation which is an element of $U^{+}$should have some special properties worth studying. Indeed, this is one of the main uses of the Ringel-Hall algebra approach in the case when $\triangle$ is of finite type, which is

Received by the editors August 3, 1998 and, in revised form, June 23, 1999.

2000 Mathematics Subject Classification. Primary 16G20, 17B37; Secondary 16G60, 17B40, 05A30, $18 \mathrm{G} 15$.

Supported by the Chinese Education Ministry, the Chinese Academy of Sciences, and the National Natural Science Foundation of China.

(C)2000 American Mathematical Society 
exactly the case of all indecomposable $A$-modules having no self-extensions, where $U^{+}$coincides with $\mathcal{H}(A)$, i.e. the subalgebra $\mathcal{C}(A)$ of $\mathcal{H}(A)$ coincides with $\mathcal{H}(A)$ ([R5]). Thus, the indecomposable $A$-module is just a natural choice of root vectors in $U^{+}$, and all indecomposable modules can be made a PBW-basis for $U^{+}$([R10]).

In general, $\mathcal{H}(A)$ is larger than $U^{+}$. Thus, in order to carry information on $A$-modules into $U^{+}$in the case of infinite type, a natural question is to determine which kinds of modules lie in $\mathcal{C}(A)$, or $U^{+}$. This question leads to an interesting observation due to Ringel (see e.g. [Z1, Theorem 2]): a stone belongs to $U^{+}$.

Recall that a stone means an indecomposable $A$-module $M$ without self-extension, i.e. $\operatorname{Ext}_{\mathrm{A}}^{1}(M, M)=0$. This is also called an exceptional module, a rigid module, a partial tilting module, a Schur module, and so on, and plays a very important role in representation theory. For more on this kind of modules see e.g. [C2], [H], [Ker], [R1].

For the case when $\triangle$ is of affine type, we have proved in $[\mathrm{ZZ}$ that an indecomposable module belongs to $\mathcal{C}(A)$, or $U^{+}$, if and only if it is a stone. The goal of the present paper is to deal with an arbitrary module, i.e. to determine all the modules $M$ (not necessarily indecomposable) with the property $[M] \in \mathcal{C}(A)$, over the path algebras of affine quivers. Our main result, Theorem 0.5 , shows that such a module turns out to be a direct sum of stones with some special property. For technical reasons, we only deal with the affine quivers, but in principal the arguments also work in the case of affine species, as remarked in the end of $\S 6$. Since only in the affine case do we have a satisfactory description of the module category, our restriction to this case seems to be reasonable.

0.2. Let $A$ be a finite-dimensional algebra over a finite field $k$, with pairwise nonisomorphic simple modules $S(1), \cdots, S(n)$. Consider the category $A$-mod of the finite-dimensional $A$-modules. Then $M$ is finite as a set for $M \in A$-mod. Let $K_{0}(A)$ be the Grothendieck group of all modules in $A$-mod modulo exact sequences. Then $K_{0}(A)$ can be identified with $\mathbb{Z}^{n}$ so that the image of $S(i)$ in $K_{0}(A)$ is $(0, \cdots, 0,1,0, \cdots, 0)$. For $M \in A$-mod, denote by $[M]$ its isoclass, and by $\operatorname{dim} M$ its image in $K_{0}(A)$, which is called the dimension vector of $M$.

Let $\mathbb{Q}$ be the field of rational numbers. By definition ( $[\mathrm{R} 2])$ the Ringel-Hall algebra $\mathcal{H}(A)$ of $A$ is a $\mathbb{Q}$-space with basis the set of isoclasses $[M]$ of all finite modules, with multiplication given by (for simplicity, we use the untwisted multiplication in this paper but all considerations hold for the twisted one introduced in [R9])

$$
[M] \cdot[N]:=\sum_{[L]} g_{M, N}^{L}[L]
$$

where the structure constant $g_{M, N}^{L}$ is the number of submodules $V$ of $L$ with $V \cong N$ and $L / V \cong M$. Then $\mathcal{H}(A)$ is an associative $\mathbb{Q}$-algebra with identity [0]. See also Mac.

By definition ( $[\mathrm{R} 4])$ the composition algebra $\mathcal{C}(A)$ of $A$ is the subalgebra of $\mathcal{H}(A)$ generated by all isoclasses of simple $A$-modules $[S(1)], \cdots,[S(n)]$. For $\mathbf{d}=$ $\left(d_{1}, \cdots, d_{n}\right) \in \mathbb{N}_{0}^{n}$, let $\mathcal{H}(A)_{\mathbf{d}}$ be the $\mathbb{Q}$-space with basis the set of $[M]$ with $\operatorname{dim} M=$ $\mathbf{d}$, and $\mathcal{C}(A)_{\mathbf{d}}=\mathcal{H}(A)_{\mathbf{d}} \cap \mathcal{C}(A)$. Then $\mathcal{H}(A)=\bigoplus_{\mathbf{d}} \mathcal{H}(A)_{\mathbf{d}}$ and $\mathcal{C}(A)=\bigoplus_{\mathbf{d}} \mathcal{C}(A)_{\mathbf{d}}$ are both $\mathbb{N}_{0}^{n}$-graded algebras, and $\mathcal{C}(A)_{\mathbf{d}}$ is the $\mathbb{Q}$-space spanned by all monomials $\left[S\left(i_{1}\right)\right] \cdots\left[S\left(i_{d}\right)\right]$ with $d=d_{1}+\cdots+d_{n}$, such that the number of occurrences of $i$ in the sequence $i_{1}, \cdots, i_{d}$ is exactly $d_{i}$ for $1 \leq i \leq n$. 
0.3. Let $A$ be in addition hereditary, i.e. $\operatorname{Ext}_{A}^{i}(-,-)$ vanishes for $i \geq 2$. Let us briefly recall some basic points of the representation theory of $A$ from [ARS], DR, [R1], [C1], which will be used throughout this paper.

Given two modules $X, Y$, define

$$
\langle X, Y\rangle=\operatorname{dim}_{k} \operatorname{Hom}_{A}(X, Y)-\operatorname{dim}_{k} \operatorname{Ext}_{\mathrm{A}}^{1}(X, Y) .
$$

Then it is clear that $\langle X, Y\rangle$ depends only on $\operatorname{dim} X$ and $\operatorname{dim} Y$, so it can be denoted by $\langle\operatorname{dim} X, \operatorname{dim} Y\rangle$, and bilinearly extended to $\mathbb{Z}^{n}$, which is called the Ringel form. Let $(-,-)$ be the symmetrization of the Ringel form, i.e.

$$
(i, j)=\langle S(i), S(j)\rangle+\langle S(j), S(i)\rangle, 1 \leq i, j \leq n .
$$

Then $(-,-)$ is a symmetrizable Cartan datum in Lusztig's sense [L3]. Conversely, given a symmetrizable Cartan datum, then there exists a finite-dimensional algebra, over any finite field $k$, such that the symmetrization of its Ringel form is exactly the given Cartan datum; see e.g. R11. In this correspondence, the finite-dimensional path algebras exactly correspond to the symmetric Cartan data.

Let $q(x)=\langle x, x\rangle$. Note that $q(x)$ is positive definite (resp. positive semi-definite but not positive definite, indefinite) if and only if the corresponding Cartan datum $(-,-)$ is finite (resp. affine, indefinite), and that $A$ is representation-finite (i.e. $A$ has only finitely many indecomposable modules) if and only if $q(x)$ is positive definite; see Ga. If $q(x)$ is positive semi-definite, but not positive definite, then we say $A$ is a tame hereditary algebra. In this case, $A$ is Morita equivalent to the path algebra of a Euclidean quiver, or the tensor algebra of a $k$-species associated with a non-simply-laced Euclidean diagram (see the list of Euclidean diagrams for example in [DR, ARS]), and by Euclidean quivers we mean the diagrams of types $\widetilde{A_{n}}, \widetilde{D_{n}}$, and $\widetilde{E_{m}}, m=6,7,8$, with arbitrary orientations, except the oriented cycle in the case of $\tilde{A_{n}}$ ), and there exists a unique minimal positive imaginary root $\mathbf{n}$ such that $\left\{z \in \mathbb{Z}^{n} \mid q(z)=0\right\}=\mathbb{Z} \mathbf{n}$.

Denote by $\tau=\mathrm{D} \operatorname{Tr}$ and $\tau^{-1}=\operatorname{TrD}$ the Auslander-Reiten translates. Then $\tau=D \operatorname{Ext}_{\mathrm{A}}^{1}(-, A)$ and $\tau^{-1}=\operatorname{Ext}_{\mathrm{A}}^{1}(D(A),-)$, where $D=\operatorname{Hom}_{k}(-, k)$. An indecomposable $A$-module $M$ is said to be preprojective (resp. preinjective) provided that there exists a positive integer $m$ such that $\tau^{m}(M)=0\left(\operatorname{resp} . \tau^{-m}(M)=0\right)$, and regular provided that $\tau^{t}(M) \neq 0$ for $t \in \mathbb{Z}$. Any $X \in A$-mod is said to be preprojective (resp. regular, preinjective) provided that every indecomposable direct summand of $X$ is so.

With indecomposable $A$-modules as vertices, and using the irreducible maps between indecomposables, we obtain the (valued) Auslander-Reiten quiver $\Gamma(A)$ of $A$; see [ARS] for details. When $A$ is of finite type, $\Gamma(A)$ has a unique connected component, in which all indecomposables are both preprojective and preinjective. Otherwise, $\Gamma(A)$ has one preprojective component which consists of all indecomposable preprojective modules, one preinjective component which consists of all indecomposable preinjective modules, and all other components are called regular components. If $P, R$, and $I$ are respectively preprojective, regular and preinjective modules, then we have

$$
\begin{aligned}
\operatorname{Hom}(R, P) & =\operatorname{Hom}(I, P)=\operatorname{Hom}(I, R)=0=\operatorname{Ext}_{A}^{1}(P, R) \\
& =\operatorname{Ext}_{A}^{1}(P, I)=\operatorname{Ext}_{A}^{1}(R, I) .
\end{aligned}
$$


From now on, consider a tame hereditary algebra $A$ over a finite field $k$. In this case, all regular modules form an extension-closed abelian subcategory of $A$-mod. The simple objects in this subcategory will be called quasi-simple modules; any indecomposable regular module $M$ is regular uniserial, and hence $M$ is uniquely written as $M=E(t)=(t) E^{\prime}$, where $t$ is the quasi-length, $E$ is the quasi-top, and $E^{\prime}$ is the quasi-socle of $M$.

All regular components of $\Gamma(A)$ are tubes of the form $T=\mathbb{Z A}_{\infty} / m$, where $m$ is called the rank of $T$; see [ARS, p. 287] or [R1 p. 113]. Any indecomposable $M$ in a tube $T$ of rank $m$ has the property $\tau^{m}(M)=M$; if $E$ is a quasi-simple in $T$, then $E_{i}=\tau^{i}(E), 1 \leq i \leq m$, are all quasi-simples in $T$, and $M=E_{i}(t)=$ $(t) E_{i+t-1}$ for a unique $i$. If $m=1$, then $T$ is called a homogeneous tube; otherwise, a non-homogeneous tube. There are no stones in a homogeneous tube; and an indecomposable $M$ in a non-homogeneous tube of rank $m$ is a stone if and only if the quasi-length of $M$ is less than $m$. Note that $\Gamma(A)$ has only finitely many non-homogeneous tubes (the ranks and the dimension vectors of the quasi-simples in these tubes have been given in [DR, Tables]), and infinitely many homogeneous tubes (the dimension vector of the unique quasi-simple in a homogeneous tube is a multiple of $\mathbf{n}$ ). Also, two indecomposable modules in two different tubes have no non-zero homomorphisms and no non-trivial extensions.

Define the defect $\partial(M)$ of a module $M$ to be $\langle\mathbf{n}, \operatorname{dim} M\rangle$. Then by [DR] an indecomposable $M$ is preprojective (resp. regular; preinjective) if and only if $\partial(M)<0$ $($ resp. $\partial(M)=0 ; \partial(M)>0)$.

0.4. For $\mathbf{d} \in K_{0}(A)=\mathbb{Z}^{n}$, define the following element in $\mathcal{H}(A)$ :

$$
r_{\mathbf{d}}=\sum_{[M]}[M], \quad \text { where } M \text { runs over all regular modules with } \operatorname{dim} M=\mathbf{d} .
$$

(Note that this is a finite sum since $k$ is a finite field. If there are no regular modules $M$ with $\operatorname{dim} M=\mathbf{d}$, then $r_{\mathbf{d}}:=0$. Set $r_{0}:=[0]$.) Then we have $r_{\mathbf{d}} \in \mathcal{C}(A)$; see Z1 Theorem 1].

Let $\mathcal{T}, \mathcal{P}$, and $\mathcal{I}$ denote the subalgebra of $\mathcal{H}(A)$ generated by all elements $r_{\mathbf{d}}$ with $\mathbf{d} \in \mathbb{N}_{0}^{n}$, by indecomposable preprojectives, and by indecomposable preinjectives, respectively. Then $\mathcal{P}$ (resp. $\mathcal{I}$ ) has a basis $[P]$ (resp. $[I]$ ), where $P$ (resp. $I$ ) runs over all preprojective (resp. preinjective) modules, and $\mathcal{T}$ is the $\mathbb{Q}$-space spanned by all elements $r_{\mathbf{d}_{1}} \cdots r_{\mathbf{d}_{m}}$, where $\mathbf{d}_{1}, \cdots, \mathbf{d}_{m} \in \mathbb{N}_{0}^{n}$, and $m \in \mathbb{N}_{0}$. Let $\mathcal{P} \cdot \mathcal{T} \cdot \mathcal{I}$ be the $\mathbb{Q}$-subspace of $\mathcal{H}(A)$ spanned by all elements $[P] \cdot r_{\mathbf{d}_{1}} \cdots r_{\mathbf{d}_{m}} \cdot[I]$, where $[P] \in \mathcal{P},[I] \in \mathcal{I}, \mathbf{d}_{1}, \cdots, \mathbf{d}_{m} \in \mathbb{N}_{0}^{n}$ and $m \in \mathbb{N}_{0}$.

Let $X=\sum c_{N}[N] \in \mathcal{H}(A)$ (resp. $\in \mathcal{C}(A)$ ). If all $N$ are regular modules for $c_{N} \neq 0$, then $X$ is called a regular element in $\mathcal{H}(A)$ (resp. in $\mathcal{C}(A)$ ).

We shall often use the following structure theorem from [Z3] on triangular decomposition for composition algebras of affine quivers. In [Z3] Proposition 1.1 below was used; it follows that we should assume $|k| \neq 2$.

Theorem 0.4. Let $A$ be the algebra of a Euclidean quiver over a finite field $k$ with $|k| \neq 2$. Then $\mathcal{C}(A)=\mathcal{P} \cdot \mathcal{T} \cdot \mathcal{I}=\mathcal{P} \otimes_{\mathbb{Q}} \mathcal{T} \otimes_{\mathbb{Q}} \mathcal{I}$. In particular, $\mathcal{T}$ is exactly the subalgebra of $\mathcal{C}(A)$ generated by all regular elements in $\mathcal{C}(A)$.

0.5. Let $C$ be an Auslander-Reiten component of $A$, and $M$ an $A$-module. By $M \in C$ we mean that every indecomposable direct summand of $M$ belongs to $C$.

A vector $\mathbf{x}=\left(x_{1}, \cdots, x_{m}\right)$ is said to be sincere if all $x_{i} \neq 0$. 
Let $T$ be a non-homogeneous tube of rank $m$ with all quasi-simple modules $E_{1}, \cdots, E_{m}$, and $M \in T$. Then $\operatorname{dim} M$ can be uniquely written as $\operatorname{dim} M=$ $c_{1} \operatorname{dim} E_{1}+\cdots+c_{m} \operatorname{dim} E_{m}$ (see Lemma 2.5). This fact permits us to define the quasi-dimension vector of $M$ to be

$$
\text { q. } \operatorname{dim} M=\left(c_{1}, \cdots, c_{m}\right) \in \mathbb{N}_{0}^{m} \text {. }
$$

Thus, an indecomposable module $N \in T$ is a stone if and only if q. $\operatorname{dim} N$ is non-sincere; and if $\mathbf{q}$. $\operatorname{dim} M$ is non-sincere, then $M$ is a direct sum of stones.

Since every module $M$ can be uniquely decomposed as $M=P \oplus R \oplus I$ with $P, R, I$ being preprojective, regular, and preinjective, respectively, we shall call $R$ the regular direct summand of $M$.

Our main result is as follows:

Theorem 0.5. Let $A$ be the path algebra of a Euclidean quiver over a finite field $k$ with $|k| \geq 4$, and $M$ an arbitrary $A$-module. Then $[M] \in \mathcal{C}(A)$ if and only if the regular direct summand $R$ of $M$ is a direct sum of modules from non-homogeneous tubes with quasi-dimension vector non-sincere. Namely, let $R=R_{1} \oplus \cdots \oplus R_{m}$ with $R_{i} \in T_{i}$, where $T_{1}, \cdots, T_{m}$ are pairwise different tubes. Then $[M] \in \mathcal{C}(A)$ if and only if all $T_{i}$ are non-homogeneous and all $\mathbf{q} . \operatorname{dim} R_{i}$ are non-sincere.

The proof will use among other things the classification of the module category of affine quivers, the structure of triangular decompositions of tame composition algebras, and the invariant subspaces of skew derivations. As an application, we can see that for the path algebra $A=k Q$, the composition algebra $\mathcal{C}(A)$ coincides with the Ringel-Hall algebra $\mathcal{H}(A)$ if and only if $Q$ is a Dynkin quiver, i.e. $A$ is representation-finite.

0.6. The paper is organized as follows. In $\S 1$ we prove two results on dimension vectors of homogeneous quasi-simples of tame path algebras over finite fields of more than 3 elements. This is the reason we need the assumption $|k| \geq 4$ in Theorem 0.5. In $\S 2$ we consider regular modules in a non-homogeneous tube with quasi-dimension vector $(1, \cdots, 1)$, by using Theorem 0.4 . We find out some skew derivations of $\mathcal{H}(A)$, such that the subalgebras $\mathcal{C}(A)$ and $\mathcal{T}$ are invariant subspaces in $\S 3$ (see Theorems 3.5 and 3.7). The regular modules in a non-homogeneous tube with quasi-dimension vector bigger than $(1, \cdots, 1)$ are studied in $\S 4$, by using the results and methods in $\S \S 2$ and 3 . $\S 5$ is devoted to discuss the homogeneous modules via Theorems 0.4 and 3.5. Reduction steps and the proof for Theorem 0.5 are given in $\S 6$. Finally, we include an application.

Most parts of this paper also hold for non-simply-laced tame hereditary algebras over any finite field. See Remark 6.4.

\section{Dimension Vectors of Homogeneous QuASI-SIMPLES}

Let $A$ be an arbitrary hereditary Artin algebra with $s$ simple modules, and let $X$ be a stone, i.e. an indecomposable $A$-module $M$ with $\operatorname{Ext}_{A}^{1}(M, M)=0$. Recall that the perpendicular category $X^{\perp}$ introduced by Geigle and Lenzing in [GL], and Schofield in [S], is the full subcategory given by

$$
X^{\perp}=\left\{M \in A-\bmod \mid \operatorname{Hom}_{A}(X, M)=0=\operatorname{Ext}_{\mathrm{A}}^{1}(X, M)\right\} .
$$

Then, by [GL] and [S], $X^{\perp}$ is equivalent to $B$ - $\bmod$, where $B$ is a hereditary Artin algebra with $s-1$ simple modules, and the functor $B$-mod $\longrightarrow A$-mod is exact and 
induces the isomorphisms on both Hom and Ext. Note that $\operatorname{End}_{A} X$ is isomorphic to $\operatorname{End}_{A} S$ for some simple $A$-module, and that $\operatorname{dim} X$ is a real root ( $\mathbb{R} 12$, Corollaries $1,2]$ ). If $A$ is the path algebra of a finite quiver without oriented cycles, then $B$ is also the path algebra of a quiver without oriented cycles - see for example $[$, Theorem 2.3]; in this case $\operatorname{End}_{A} X$ is always the base field, and the existence of a stone with a fixed dimension vector does not depend on the base field; see [HHKU] and also $\mathrm{K} 2$ K3.

The aim of this section is to prove Propositions 1.1 and 1.4 for latter applications. It seems that they are also of independent interest.

For a set $X$, denote by $|X|$ the cardinal of $X$.

Proposition 1.1. Let $A$ be the path algebra of a Euclidean quiver over an arbitrary field $k$, with minimal positive imaginary root $\mathbf{n}$. Denote by $t$ the number of homogeneous quasi-simple modules with dimension vector $\mathbf{n}$. Then:

(i) For the Kronecker algebra, $t=|k|+1$.

(ii) For $\tilde{A_{n}}(n \geq 2)$ with $n_{1}$ arrows going clockwise and $n_{2}$ arrows going anticlockwise, if $n_{1}, n_{2} \geq 2$, then $t=|k|-1$; if $n_{1}=1$ or $n_{2}=1$, then $t=|k|$.

(iii) For $\widetilde{D_{n}}$ and $\tilde{E}_{n}$, we have $t=|k|-2$.

In particular, if $|k| \neq 2$, then there always exists at least one homogeneous quasisimple module with dimension vector $\mathbf{n}$; and if $|k|=2$, then for type $\tilde{A_{n}}$ there exists at least one homogeneous quasi-simple module with dimension vector $\mathbf{n}$, and for types $\tilde{D}_{n}$ and $\tilde{E}_{n}$ there exist no homogeneous quasi-simple modules with dimension vector $\mathbf{n}$.

Before the proof we recall the following easy fact

Lemma 1.2. Let $A$ be a hereditary Artin algebra, and let $X$ be an A-stone. Let $B$ be the hereditary Artin algebra with $B$-mod equivalent to $X^{\perp}$. Let $M \in X^{\perp}$ with $\operatorname{dim} M=\left(d_{1}, \cdots, d_{m}\right)$ as a B-module. Then $\operatorname{dim} M=d_{1} \operatorname{dim} E(1)+\cdots+$ $d_{m} \operatorname{dim} E(m)$ as an $A$-module, where $E(1), \cdots, E(m)$ are all non-isomorphic simple B-modules. In particular, if $M, N \in X^{\perp}$ with $\operatorname{dim} M=\operatorname{dim} N$ as B-modules, then $\operatorname{dim} M=\operatorname{dim} N$ as A-modules.

Proof. This follows from the definition of dimension vectors.

1.3. Proof of Proposition 1.1. By $q$ we denote $|k|$, and by $s$ the number of the simple $A$-modules.

If $s=2$, then $A$ is the Kronecker algebra and obviously $t=q+1$.

If $s=3$, then $A$ is of type $\tilde{A_{2}}$. Let $E\left(\lambda_{1}, \lambda_{2}, \lambda_{3}\right)=\left(k_{1}, k_{2}, k_{3}\right)$ with $k_{1}=k_{2}=$ $k_{3}=k$, and $k$-maps $\lambda_{1}: k_{1} \longrightarrow k_{2}, \lambda_{2}: k_{2} \longrightarrow k_{3}$ and $\lambda_{3}: k_{1} \longrightarrow k_{3}$. Then it is clear that $E\left(\lambda_{1}, \lambda_{2}, \lambda_{3}\right)$ is indecomposable if and only if all $\lambda_{i} \neq 0$, or only one $\lambda_{i}=0$; and that if $\lambda_{1} \lambda_{2} \lambda_{3} \mu_{1} \mu_{2} \mu_{3} \neq 0$, then $E\left(\lambda_{1}, \lambda_{2}, \lambda_{3}\right) \simeq E\left(\mu_{1}, \mu_{2}, \mu_{3}\right)$ if and only if $\lambda_{1} \lambda_{2} / \lambda_{3}=\mu_{1} \mu_{2} / \mu_{3}$. In this way we get $(q-1)+3=q+2$ pairwise indecomposable regular modules with dimension vector $(1,1,1)$. Since $A$ has a unique non-homogeneous tube, which is of rank 2 , it follows that there are $q$ homogeneous quasi-simples with dimension vector $(1,1,1)$.

If $A$ is of type $\tilde{A_{3}}$ with two arrows going clockwise and two arrows going anticlockwise, then $A$ has 2 non-homogeneous tubes $T$ and $U$, both of rank 2 . In order to count $t$, the number of homogeneous quasi-simple modules with dimension vector $(1,1,1,1)$, one can for example take a quasi-simple stone $X$ in $T$, with 
$X^{\perp}$ equivalent to $B$-mod. Then $B$ is of type $\tilde{A_{2}}$. Take an indecomposable $A$ module $M$ in $U$ with $\operatorname{dim} M=(1,1,1,1)$. Then the whole tube $U$ is in $X^{\perp}$, it becomes naturally a tube of $B$, and hence $\operatorname{dim} M=(1,1,1)$ as a $B$-module. It follows from Lemma 1.2 that any indecomposable regular $A$-module $N \in X^{\perp}$ with $\operatorname{dim} N=(1,1,1,1)$ is of dimension vector $(1,1,1)$ as a $B$-module: Indeed, notice that $N$ as a $B$-module is homogeneous quasi-simple or is of quasi-length 2 . Since $N$ is not a stone and $\operatorname{End}_{B} N=\operatorname{End}_{A} N$ is a field, it follows that as a $B$-module $\operatorname{dim} N$ is a multiple of $(1,1,1)$, and hence by Lemma 1.2 we see that it must be $(1,1,1)$ since $\operatorname{dim}_{B} M=(1,1,1)$. In this way we see that

$$
t+3=q+2
$$

this proves $t=q-1$.

Using this argument, we can reduce any case to $\tilde{A_{2}}$. Note that by the tables in DR the perpendicular category of quasi-simple stones is known. This completes the proof.

Proposition 1.4. Let $A$ be the path algebra of a Euclidean quiver over a finite field $k$ with $|k| \geq 4$. Let $E_{1}$ be a homogeneous quasi-simple A-module. Then there exists a homogeneous quasi-simple $A$-module $E_{2}$ with $E_{2} \not E_{1}$ and $\operatorname{dim} E_{1}=\operatorname{dim} E_{2}$.

For the proof we need the following:

Lemma 1.5. Let $A$ be the path algebra of a Euclidean quiver over an arbitrary field, and $T$ a tube of $A$ with rank bigger than 2 , and let $X$ be a quasi-simple stone in $T$ with $X^{\perp}$ equivalent to B-mod. If $E$ is a homogeneous quasi-simple B-module, then $E$ is also a homogeneous quasi-simple A-module.

Proof. Note that $E$ is clearly regular as an $A$-module. Since $\operatorname{End}_{A} E=\operatorname{End}_{B} E$ is a field and $\operatorname{Ext}_{A}^{1}(E, E)=\operatorname{Ext}_{B}^{1}(E, E) \neq 0$, it follows that as an $A$-module $E$ is either a homogeneous quasi-simple $A$-module or an indecomposable regular module lying in a non-homogeneous tube of rank $m$ with quasi-length $m$. We claim that $E$ must be a homogeneous quasi-simple $A$-module.

Otherwise, $E$ is an indecomposable regular $A$-module lying in a non-homogeneous tube of rank $m$ with quasi-length $m$. Then $E$ must be in $T$, since $E$ is a homogeneous quasi-simple $B$-module. Consider the quasi-socle $M$ of $E$ as an $A$-module. Note that $M \neq X$, since $\operatorname{Hom}(X, E)=0$.

If $M \neq \tau X$, then $M \in X^{\perp}$. Note that $M$ is also a regular $B$-module. (In order to see this, consider all indecomposable $A$-modules $Y$ with quasi-top $M$; then $\operatorname{Ext}_{A}^{1}(X, Y)=\operatorname{Hom}(Y, \tau X)=0$ since $M \neq \tau X$. Note that $\operatorname{Hom}(X, Y) \neq 0$ for those $Y$ whose quasi-socles are not $X$. In this way we get infinitely many indecomposable $A$-modules $Y$ such that $Y \in X^{\perp}$ and $\operatorname{Hom}(Y, M) \neq 0$, and it follows that $M$ cannot be a preprojective $B$-module. Dually, $M$ cannot be a preinjective $B$-module.) Thus we have $\operatorname{Hom}_{B}(M, E)=\operatorname{Hom}_{A}(M, E) \neq 0$, and $\operatorname{dim}_{k} M<\operatorname{dim}_{k} E$. This contradicts that $E$ is a quasi-simple $B$-module.

If $M=\tau X$, then $\tau M \neq X$ since the rank of $T$ is bigger than 2. Consider the quasi-top $M^{\prime}$ of $E$ as an $A$-module. Note that $M^{\prime}=\tau M, M^{\prime} \in X^{\perp}$, and $M^{\prime}$ is also a regular $B$-module. (In order to see this, consider all indecomposable $A$-modules $Y$ with quasi-top $M^{\prime}$; then $\operatorname{Ext}_{A}^{1}(X, Y)=\operatorname{Hom}(Y, \tau X)=0$ since $M^{\prime} \neq \tau X$. Note that $\operatorname{Hom}(X, Y)=0$ for those $Y$ whose quasi-socles are not $X$. In this way we get infinitely many indecomposable $A$-modules $Y$ such that $Y \in X^{\perp}$ and $\operatorname{Hom}\left(Y, M^{\prime}\right) \neq$ 0 . It follows that $M^{\prime}$ cannot be a preprojective $B$-module. Dually, $M$ is not a 
preinjective $B$-module.) Thus we have $\operatorname{Hom}_{B}\left(E, M^{\prime}\right)=\operatorname{Hom}_{A}\left(E, M^{\prime}\right) \neq 0$, and $\operatorname{dim}_{k} M^{\prime}<\operatorname{dim}_{k} E$, which contradicts that $E$ is a quasi-simple $B$-module. This completes the proof.

1.6. Recall the following fact: if $k$ is a finite field with $|k|=q \neq 2$, then the number $N(q, n)$ of monic irreducible polynomials in $k[x]$ of degree $n$ is bigger than 1 . Note that $N(2,2)=1$.

In fact, by a well-known formula of Gauss (see, e.g. [J p.289])

$$
n N(q, n)=\sum_{d \mid n} \mu\left(\frac{n}{d}\right) q^{d},
$$

where $\mu$ is the Möbius function. It follows that $N(q, n) \geq 1$ for all $q, n$; and $N(q, n)=1$ if and only if $q=n=2$.

In the case of the Kronecker algebra we can prove even more.

Lemma 1.7. Let $K$ be the Kronecker algebra over a finite field $k$ with $|k| \neq 2$. Then for every positive integer $\lambda$, there exist at least two non-isomorphic quasisimple $K$-modules $E_{1}$ and $E_{2}$ such that $\operatorname{dim} E_{1}=\operatorname{dim} E_{2}=\lambda(1,1)$.

Proof. Let $F$ be the finite field with $[F: k]=\lambda$. By the preceding fact in 1.6 there are two different monic irreducible polynomials $p_{1}(x) \neq p_{2}(x)$ of degree $\lambda$ in $k[x]$. Consider $K$-modules: $E_{i}=\left(k[x] /\left\langle p_{i}(x)\right\rangle \rightrightarrows k[x] /\left\langle p_{i}(x)\right\rangle\right)$, i.e. $E_{i}=(F, F)$ with two $k$-linear transformations given by identity and multiplication by $x+\left\langle p_{i}(x)\right\rangle$. Clearly we have $\operatorname{End}_{K} E_{i}=F$ for $i=1,2$, and it follows that both $E_{1}$ and $E_{2}$ are quasi-simple $K$-modules with dimension vector $\lambda(1,1)$. Since $p_{1}(x) \neq p_{2}(x)$, it is easily seen that $E_{1}$ and $E_{2}$ are non-isomorphic.

Now, we investigate three special cases, for which all non-homogeneous tubes are of rank 2, and hence Lemma 1.5 cannot be applied.

Lemma 1.8. Let $A$ be the $k$-path algebra of type $\tilde{A_{2}}$ with $|k| \neq 2$. Let $E_{1}$ be a homogeneous quasi-simple A-module. Then there exists a homogeneous quasisimple A-module $E_{2}$ with $E_{2} \nsucceq E_{1}$ and $\operatorname{dim} E_{1}=\operatorname{dim} E_{2}$.

Proof. Let $X$ be a quasi-simple in the unique non-homogeneous tube $T$ of $A$. Note that $T$ is of rank 2 and $X^{\perp}$ is the module category of the Kronecker algebra $K$. Since $E_{1} \in X^{\perp}$ and $E_{1}$ is also a homogeneous quasi-simple $K$-module, it follows from Lemma 1.7 that there exists a (homogeneous) quasi-simple $K$-module $E_{2}$ such that $E_{1} \nsucceq E_{2}$ and $\operatorname{dim} E_{1}=\operatorname{dim} E_{2}$ as $K$-modules. It follows from Lemma 1.2 that $\operatorname{dim} E_{1}=\operatorname{dim} E_{2}$ as $A$-modules. If $E_{2}$ is also a homogeneous quasi-simple $A$-module, then the assertion is proved. If it is not, then $E_{2} \in T$, and $E_{2}$ is of quasi-length 2 since End ${ }_{A} E_{2}$ is a field and $E_{2}$ is not an $A$-stone. It follows that $\operatorname{dim} E_{2}=(1,1,1)$, and hence $\operatorname{dim} E_{1}=(1,1,1)$. Then the assertion follows from Proposition 1.1. This completes the proof.

With the same argument we can prove the following two lemmas.

Lemma 1.9. Let $A$ be the $k$-path algebra of type $\widetilde{A_{3}}$ with $|k| \geq 3$. Let $E_{1}$ be a homogeneous quasi-simple A-module. Then there exists a homogeneous quasisimple A-module $E_{2}$ with $E_{2} \nsucceq E_{1}$ and $\operatorname{dim} E_{1}=\operatorname{dim} E_{2}$. 
Lemma 1.10. Let $A$ be the $k$-path algebra of type $\widetilde{D}_{4}$ with $|k| \geq 4$. Let $E_{1}$ be a homogeneous quasi-simple A-module. Then there exists a homogeneous quasi-simple $A$-module $E_{2}$ with $E_{2} \nsucceq E_{1}$ and $\operatorname{dim} E_{1}=\operatorname{dim} E_{2}$.

1.11. Proof of Proposition 1.4. By Lemmas 1.7 and 1.8 we may assume that the number of the simple $A$-modules is $s \geq 4$.

If there exists a non-homogeneous tube $T$ of rank bigger than 2 , then choose a quasi-simple stone $N \in T$. Let $B$ be the path algebra with $s-1$ simple modules such that $B$-mod is equivalent to $N^{\perp}$. Note that $E_{1} \in N^{\perp}$ and $E_{1}$ is also a homogeneous quasi-simple $B$-module. Then by induction there exists a homogeneous quasi-simple $B$-module $E_{2}$ with $E_{2} \not 千 E_{1}$ and $\operatorname{dim} E_{2}=\operatorname{dim} E_{1}$ as $B$-modules. It follows from Lemma 1.2 that $\operatorname{dim} E_{1}=\operatorname{dim} E_{2}$ as $A$-modules, and from Lemma 1.5 that $E_{2}$ is also a homogeneous quasi-simple $A$-module.

If all non-homogeneous tubes are of rank 2 , then $A$ is of type $\widetilde{A}_{3}$, or $\widetilde{D}_{4}$, and then the assertion follows from Lemmas 1.9 and 1.10. This completes the proof.

\section{Modules With QUASI-Dimension VeCtor $(1, \cdots, 1)$ IN A NON-HOMOGENEOUS TUBE}

The aim of this section is to prove the following fact.

Proposition 2.1. Let $A$ be the $k$-path algebra of a Euclidean quiver with $|k| \neq 2$, let $T$ be a non-homogeneous tube of $A$, and let $M$ be an arbitrary A-module in $T$ with q. $\operatorname{dim} M=(1, \cdots, 1)$. Then $[M] \notin \mathcal{C}(A)$.

In order to prove Proposition 2.1, we first prove the following special case of $M$ indecomposable.

Lemma 2.2. Let $A$ be the $k$-path algebra of a Euclidean quiver with $|k| \neq 2$, let $T$ be a non-homogeneous tube of rank $m$, and let $N$ be an indecomposable module of quasi-length $m$ in $T$. Then $[N] \notin \mathcal{C}(A)$.

Lemma 2.2 has been used in [ZZ, Lemma 2.1] (however, the condition $|k| \neq 2$ is not indicated explicitly there). Since we want to indicate here which parts in the proof are also valid for non-simply laced tame hereditary algebras, and why $|k| \neq 2$ is needed, we include a proof below.

Note that when $A$ is representation-finite some of the structure constants of $\mathcal{H}(A)$ can be given by the Hall polynomials; see [R3]. For a finite $A$-module $M$, denote by $a_{M}$ the order of the automorphism group of $M$ as an $A$-module. The following facts will be used often.

Lemma 2.3. Let $A$ be an arbitrary finite-dimensional algebra over a finite field $k$ with $q$ elements, and let $M, N$ be A-modules.

(i) Assume that $\operatorname{Hom}_{A}(N, M)=0$. If $g_{M, N}^{L} \neq 0$, then $g_{M, N}^{L}=1$.

(ii) Assume that $\operatorname{dim}_{k} \operatorname{Ext}_{\mathrm{A}}^{1}(M, N)=1$. If $\eta: 0 \longrightarrow N \longrightarrow L \longrightarrow M \longrightarrow 0$ is a non-split exact sequence, then

$$
[M] \cdot[N]=\frac{(q-1) a_{L}}{a_{M} a_{N}\left|\operatorname{Hom}_{A}(M, N)\right|}[L]+\frac{a_{M \oplus N}}{a_{M} a_{N}\left|\operatorname{Hom}_{A}(M, N)\right|}[M \oplus N] .
$$

(iii) Assume that $F=\operatorname{End}_{A} M$ is a field with $[F: k]=m$ and that

$$
\operatorname{dim}_{F} \operatorname{Ext}_{A}^{1}(M, N)=1 .
$$


If $\eta: 0 \longrightarrow N \longrightarrow L \longrightarrow M \longrightarrow 0$ is a non-split exact sequence, then

$$
[M] \cdot[N]=\frac{a_{L}}{a_{N}\left|\operatorname{Hom}_{A}(M, N)\right|}[L]+\frac{a_{M \oplus N}}{\left(q^{m}-1\right) a_{N}\left|\operatorname{Hom}_{A}(M, N)\right|}[M \oplus N] .
$$

(iii') Assume that $F=\operatorname{End}_{A} N$ is a field with $[F: k]=m$ and that

$$
\operatorname{dim}_{F} \operatorname{Ext}_{A}^{1}(M, N)=1 \text {. }
$$

If $\eta: 0 \longrightarrow N \longrightarrow L \longrightarrow M \longrightarrow 0$ is a non-split exact sequence, then

$$
[M] \cdot[N]=\frac{a_{L}}{a_{M}\left|\operatorname{Hom}_{A}(M, N)\right|}[L]+\frac{a_{M \oplus N}}{\left(q^{m}-1\right) a_{M}\left|\operatorname{Hom}_{A}(M, N)\right|}[M \oplus N] .
$$

Note that the assertion (i) is easy to see; for the proof of (ii), see e.g. [Z1, p.166], and (iii) and (iii') can be similarly proved.

In the following subsections $2.4-2.7$, let $A$ be an arbitrary tame hereditary algebra (not necessarily the path algebra) over any finite field. Let $T$ be a nonhomogeneous tube in the Auslander-Reiten quiver of $A$, with all quasi-simple modules $E_{1}, \cdots, E_{m}$ such that $\tau E_{i}=E_{i+1}, 1 \leq i \leq m-1$, and $\tau E_{m}=E_{1}$. Then we have $\operatorname{dim} E_{1}+\cdots+\operatorname{dim} E_{m}=\lambda \mathbf{n}$, where $\lambda$ is a positive integer with $\lambda \leq g$, where $g$ is the tier number of the valued diagram of $A$-see [M] and [DR. Tables] for the definition and the values of $g$. In particular, we have $1 \leq g \leq 3$, and for the Euclidean quivers we have $g=1$. Let $E_{i}(t)$ denote the indecomposable module in $T$ with quasi-top $E_{i}$ and quasi-length $t$.

2.4. Denote by $\mathcal{H}(T)$ the subalgebra of $\mathcal{H}(A)$ generated by all elements $[M]$ with $M \in T$, and denote by $\mathcal{C}(T)$ the subalgebra of $\mathcal{H}(T)$ generated by all quasi-simple modules in $T$. Note that both $\mathcal{H}(T)$ and $\mathcal{C}(T)$ are $\mathbb{N}_{0}^{n}$-graded algebras, and that the homogeneous component $\mathcal{H}(T)_{\mathbf{d}}$ is the $\mathbb{Q}$-space with basis the set of isoclasses $[M]$ with $M \in T$ and $\operatorname{dim} M=\mathbf{d}$, and $\mathcal{C}(T)_{\mathbf{d}}=\mathcal{H}(T)_{\mathbf{d}} \cap \mathcal{C}(T)$. Since all quasi-simple modules in $T$ are stones, it follows from a theorem due to Ringel (i.e. a stone lies in $\mathcal{C}(A)$, see e.g. [Z1, Theorem 2]) that $\mathcal{C}(T) \subseteq \mathcal{C}(A)$.

Let $\mathbf{x}=\left(x_{1}, \cdots, x_{n}\right), \mathbf{y}=\left(y_{1}, \cdots, y_{n}\right) \in \mathbb{N}_{0}^{n}$. By $\mathbf{x} \leq \mathbf{y}$ we mean $x_{i} \leq y_{i}$ for $1 \leq i \leq n$, and $\mathbf{x}<\mathbf{y}$ if $\mathbf{x} \leq \mathbf{y}, \mathbf{x} \neq \mathbf{y}$.

Proposition. Let $T$ be a non-homogeneous tube of $A$ such that the sum of dimension vectors of all quasi-simple modules in $T$ is $\lambda \mathbf{n}$ with $\lambda$ a positive integer. If $\mathbf{d} \in \mathbb{N}_{0}^{n}$ and $\mathbf{d} \not \lambda \mathbf{n}$, then $\mathcal{H}(T)_{\mathbf{d}}=\mathcal{C}(T)_{\mathbf{d}} \subseteq \mathcal{C}(A)$.

Proof. Let $N=E_{1}(t)$ be a stone which is not a quasi-simple module. Then $2 \leq$ $t \leq m-1$. Since $\operatorname{dim}_{F} \operatorname{Ext}_{A}^{1}\left(E_{1}, E_{2}(t-1)\right)=1$ with $F=\operatorname{End}_{A} E_{1}$ a field, and $\operatorname{Hom}_{A}\left(E_{2}(t-1), E_{1}\right)=\operatorname{Ext}_{A}^{1}\left(E_{2}(t-1), E_{1}\right)=\operatorname{Hom}_{A}\left(E_{1}, E_{2}(t-1)\right)=0$, it follows from Lemma 2.3 that

$$
[N]=\left[E_{1}\right] \cdot\left[E_{2}(t-1)\right]-\left[E_{2}(t-1)\right] \cdot\left[E_{1}\right] .
$$

Thus, by induction on $t$ we see that $[N]$ is an iterated commutator of the quasisimple modules in $T$, and hence $[N] \in \mathcal{C}(T)$. Let $M$ be an arbitrary module in $T$ with $\operatorname{dim} M=\mathbf{d} \not \lambda \mathbf{n}$. Then $M=M_{1} \oplus \cdots \oplus M_{m}$, with all $M_{i}$ being stones. Use induction on $\operatorname{dim}_{k} \operatorname{Ext}_{A}^{1}(M, M)$. If $\operatorname{dim}_{k} \operatorname{Ext}_{A}^{1}(M, M)=0$, then $[M]=$ $c\left[M_{1}\right] \cdots\left[M_{m}\right]$ with $c$ a non-zero number, and it follows that $[M] \in \mathcal{C}(T)$ since all $\left[M_{i}\right] \in \mathcal{C}(T)$ by the above argument. Let $\operatorname{dim}_{k} \operatorname{Ext}_{A}^{1}(M, M) \neq 0$. Then $M$ must be decomposable. Let $\left[M_{1}\right] \cdots\left[M_{m}\right]=c_{M}[M]+\sum_{[N] \neq[M]} c_{N}[N]$. Then $c_{M} \neq 0$, and for every $N$ with $c_{N} \neq 0$ we have $\operatorname{dim}_{k} \operatorname{Ext}_{A}^{1}(N, N)<\operatorname{dim}_{k} \operatorname{Ext}_{A}^{1}(M, M)$ 
(see e.g. $\mathrm{GP}$ Prop. 2.1]). Hence $[N] \in \mathcal{C}(T)$ by induction, and it follows that $[M] \in \mathcal{C}(T) \subseteq \mathcal{C}(A)$.

2.5. Let $T_{s}, 1 \leq s \leq t$, be all non-homogeneous tubes of $A$, and let $n_{s}$ be the rank of $T_{s}$. Let $e_{1}^{(s)}, \cdots, e_{n_{s}}^{(s)}$ be the dimension vectors of all quasi-simple modules in $T_{s}$, and let

$$
\operatorname{Ker} \partial=\left\{x \in \mathbb{N}_{0}^{n} \mid \partial(x)=\langle\mathbf{n}, x\rangle=0\right\} .
$$

From the table in [DR] (and also [R1 p. 146]) we have the following.

Lemma $([\overline{\mathrm{DR}}])$. The elements $\mathbf{n}$ and $e_{i}^{(s)}, 2 \leq i \leq n_{s}, 1 \leq s \leq t$, form a $\mathbb{Z}$-basis of Ker $\partial$.

Notation. Let $X=\sum_{[M]} c_{M}[M] \in \mathcal{H}(A)$. If $c_{M} \neq 0$, then $[M]$ is said to be a component of $X$ with coefficient $c_{M}$. If $[M]$ is a component of $X$ with $M$ indecomposable (resp. with $M$ indecomposable and $M \in T$, where $T$ is a tube), then $[M]$ is said to be an indecomposable component of $X$ (resp. an indecomposable $T$-component of $X$ ).

Lemma 2.6. Let $\mathbf{d} \in \mathbb{N}_{0}^{n}$ and $\mathbf{d} \supsetneqq \lambda \mathbf{n}$, and let $T$ be a non-homogeneous tube with the sum of dimension vectors of all quasi-simple modules in $T$ being $\lambda \mathbf{n}$. If $r_{\mathbf{d}}$ has a $T$-component, then $r_{\mathbf{d}} \in \mathcal{C}(T)_{\mathbf{d}}$.

Proof. By Proposition 2.4 it suffices to prove $r_{\mathbf{d}} \in \mathcal{H}(T)_{\mathbf{d}}$, i.e. any regular module with dimension vector $\mathbf{d}$ is in $T$.

Assume that $L$ is a regular module with $\operatorname{dim} L=\mathbf{d}$ and $L \notin T$. Let $L=L_{1} \oplus L_{2}$ with $L_{1} \in T$ and $0 \neq L_{2}$ having no direct summands in $T$. Let $M$ be a module with $M \in T$ and $\operatorname{dim} M=\mathbf{d}$. Then of course $\operatorname{dim} M-\operatorname{dim} L_{1}$ is an $\mathbb{N}$-combination of the dimension vectors of some quasi-simple modules in $T$. Since the sum of dimension vectors of all quasi-simple modules in $T$ is $\lambda \mathbf{n}$, and $\operatorname{dim} M-\operatorname{dim} L_{1}=\operatorname{dim} L_{2}<$ $\mathbf{d} \not \lambda \mathbf{n}$, it follows that we get a contradiction by Lemma 2.5 (note that by Lemma 2.5 the elements $e_{i}^{(s)}, 2 \leq i \leq n_{s}, 1 \leq s \leq t$, are $\mathbb{Z}$-linearly independent).

2.7. In this subsection we shall investigate the homogeneous component $\mathcal{C}(T)_{\lambda \mathbf{n}}$ of the graded algebra $\mathcal{C}(T)$, where $\lambda \mathbf{n}$ is the sum of dimension vectors of all quasisimple modules in $T$. We will present a linearly generating set of $\mathcal{C}(T)_{\lambda \mathbf{n}}$, which turns out to be useful in determining the modules in $\mathcal{C}(T)_{\lambda \mathbf{n}}$.

Let $F$ be the field $\operatorname{End}_{A} E_{i}$. Then

$$
\operatorname{dim}_{F} \operatorname{Ext}_{A}^{1}\left(E_{i}, E_{j}\right)= \begin{cases}1, & j=i+1 \\ 0, & \text { otherwise }\end{cases}
$$

Set $\mathbf{d}_{i}=\lambda \mathbf{n}-\operatorname{dim} E_{i}, 1 \leq i \leq m$. Then by definition we see that

$$
\mathcal{C}(T)_{\lambda \mathbf{n}}=\left[E_{1}\right] \cdot \mathcal{C}(T)_{\mathbf{d}_{1}}+\cdots+\left[E_{m}\right] \cdot \mathcal{C}(T)_{\mathbf{d}_{m}}
$$

According to Proposition 2.4 we see that $\mathcal{C}(T)_{\mathbf{d}_{i}}=\mathcal{H}(T)_{\mathbf{d}_{i}}$ has a basis $[L]$, where $L$ runs over modules in $T$ with dimension vector $\mathbf{d}_{i}$. Thus, $\mathcal{C}(T)_{\lambda \mathbf{n}}$ is a $\mathbb{Q}$-vector space spanned by elements in $\Omega$, where

$$
\Omega=\left\{\left[E_{i}\right] \cdot[L] \mid 1 \leq i \leq m, L \in T, \operatorname{dim} L=\mathbf{d}_{i}\right\} .
$$


Take an element $\left[E_{i}\right] \cdot[L] \in \Omega$. Since $\operatorname{dim} L=\sum_{j \neq i} \operatorname{dim} E_{j}$, it follows from the structure of a tube that $\operatorname{Hom}_{A}\left(E_{i}, L\right)=0$, and hence

$$
\begin{aligned}
\operatorname{dim}_{k} \operatorname{Ext}_{\mathrm{A}}^{1}\left(E_{i}, L\right) & =-\left\langle\operatorname{dim} E_{i}, \mathbf{d}_{i}\right\rangle=\left\langle\operatorname{dim} E_{i}, \operatorname{dim} E_{i}-\lambda \mathbf{n}\right\rangle \\
& =\left\langle\operatorname{dim} E_{i}, \operatorname{dim} E_{i}\right\rangle=\operatorname{dim}_{k} \operatorname{Hom}_{A}\left(E_{i}, E_{i}\right) .
\end{aligned}
$$

It follows that $\operatorname{dim}_{F} \operatorname{Ext}_{A}^{1}\left(E_{i}, L\right)=1$. From this fact, the structure of a tube, and the fact that the quasi-dimension vector of $L$ is $\epsilon_{1}+\cdots+\epsilon_{i-1}+\epsilon_{i+1}+\cdots+\epsilon_{m}$, where $\epsilon_{i}=$ q. $\operatorname{dim} E_{i}=(0, \cdots, 0,1,0, \cdots, 0)$, it follows that $L$ is of the following form:

$$
\begin{aligned}
L & =E_{i+1}\left(v_{1}\right) \oplus E_{i+v_{1}+1}\left(v_{2}\right) \oplus \cdots \oplus E_{i+v_{1}+\cdots+v_{t-1}+1}\left(v_{t}\right) \\
& =E_{i+1}\left(v_{1}\right) \oplus L^{\prime}
\end{aligned}
$$

where $v_{1}, \cdots, v_{t} \geq 1 ; t \geq 1 ; v_{1}+v_{2}+\cdots+v_{t}=m-1$. Since $\operatorname{dim}_{F} \operatorname{Ext}_{A}^{1}\left(E_{i}, L\right)=1$ and $\operatorname{Hom}_{A}\left(L, E_{i}\right)=0$, it follows from Lemma 2.3 that

$$
\left[E_{i}\right] \cdot[L]=\left[E_{i} \oplus L\right]+\left[E_{i}\left(v_{1}+1\right) \oplus L^{\prime}\right] .
$$

Notation. Denote by $d(M)$ the number of indecomposable direct summands of a module $M$. Thus, $d(L)=t$, and $d\left(L^{\prime}\right)=t-1$. In 2.9 below we shall call $d(M)$ the decomposition number of the element $[M]$.

Now, consider the two components of the element $\left[E_{i}\right] \cdot[L] \in \Omega$; they are $\left[E_{i} \oplus L\right]$ and $\left[E_{i}\left(v_{1}+1\right) \oplus L^{\prime}\right]$ as indicated in (2). There are $t+1$ elements in $\Omega$, which have the component $\left[E_{i} \oplus L\right]$; they are as follows (denote by $M_{j}$ the $j$-th indecomposable direct summand of $L$, namely, $\left.E_{i+v_{1}+\cdots+v_{j-1}+1}\left(v_{j}\right), 1 \leq j \leq t\right)$ :

$$
\begin{aligned}
& {\left[E_{i}\right] \cdot[L] ;} \\
& {\left[E_{i+v_{1}+\cdots+v_{j}+1}\right] \cdot\left[L_{j}\right], \quad 0 \leq j \leq t-1,}
\end{aligned}
$$

where

$$
L_{j}=E_{i} \oplus\left(\bigoplus_{\substack{1 \leq l \leq t \\ l \neq j+1}} M_{l}\right) \oplus E_{i+v_{1}+\cdots+v_{j}+2}\left(v_{j+1}-1\right) .
$$

And, there are $t$ elements in $\Omega$, which have the component $\left[E_{i}\left(v_{1}+1\right) \oplus L^{\prime}\right]$; they are as follows:

$$
\begin{aligned}
& {\left[E_{i}\right] \cdot[L] ;} \\
& {\left[E_{i+v_{1}+\cdots+v_{j}+1}\right] \cdot\left[L_{j}^{\prime}\right], \quad 1 \leq j \leq t-1,}
\end{aligned}
$$

where (note that $M_{j+1}$ is the $j$-th indecomposable direct summand of $L^{\prime}, 1 \leq j \leq$ $t-1)$

$$
L_{j}^{\prime}=E_{i}\left(v_{1}+1\right) \oplus\left(\bigoplus_{\substack{2 \leq l \leq t \\ l \neq j+1}} M_{l}\right) \oplus E_{i+v_{1}+\cdots+v_{j}+2}\left(v_{j+1}-1\right) .
$$

In the rest of this section we come back to the case of $A$ being the $k$-path algebra of a Euclidean quiver with $|k| \neq 2$.

Claim 2.8. Let $T$ be a non-homogeneous tube of rank $m$, and $N$ an indecomposable module of quasi-length $m$ in $T$. If $[N] \in \mathcal{C}(A)$, then $[N] \in \mathcal{C}(T)$. 
Proof. Assume that $[N] \in \mathcal{C}(A)$. It follows from Theorem 0.4 that $[N] \in \mathcal{T}$, where $\mathcal{T}$ is the subalgebra of $\mathcal{C}(A)$ generated by all elements $r_{\mathbf{d}}, \mathbf{d} \in \mathbb{N}_{0}^{n}$. Thus, $[N]$ can be expressed as (note that here $A$ is the path algebra and hence $\operatorname{dim} N=\mathbf{n}$ )

$$
[N]=c r_{\mathbf{n}}+\sum c_{\mathbf{d}_{1}, \cdots, \mathbf{d}_{s}} r_{\mathbf{d}_{1}} \cdots r_{\mathbf{d}_{s}}=c r_{\mathbf{n}}+X,
$$

where each summand $c_{\mathbf{d}_{1}, \cdots, \mathbf{d}_{s}} r_{\mathbf{d}_{1}} \cdots r_{\mathbf{d}_{s}} \neq 0, \mathbf{d}_{1}, \cdots, \mathbf{d}_{s} \in \mathbb{N}_{0}^{n}-\{0\}, \mathbf{d}_{1}+\cdots+\mathbf{d}_{s}=$ $\mathbf{n}, s \geq 2$, and $c, c_{\mathbf{d}_{1}, \cdots, \mathbf{d}_{s}} \in \mathbb{Q}$. Since $|k| \geq 3$, by Proposition 1.1 there exists a homogeneous quasi-simple module $E$ with $\operatorname{dim} E=\mathbf{n}$. Note that $[E]$ is not a component of $X$, since $E$ has no (non-zero) regular submodules other than $E$ and since by assumption $s \geq 2, \mathbf{d}_{i}<\mathbf{n}, 1 \leq i \leq s$. However, $[E]$ is a component of $r_{\mathbf{n}}$. Now, by comparing the coefficients of $[E]$ in both sides of the equality $(*)$ we get $c=0$ in $(*)$. Let $c_{\mathbf{d}_{1}, \cdots, \mathbf{d}_{s}} r_{\mathbf{d}_{1}} \cdots r_{\mathbf{d}_{s}}$ be a summand of $X$ having a $T$-component. Then all elements $r_{\mathbf{d}_{1}}, \cdots, r_{\mathbf{d}_{s}}$ should have a $T$-component. It follows from Lemma 2.6 that $r_{\mathbf{d}_{1}}, \cdots, r_{\mathbf{d}_{s}} \in \mathcal{C}(T)$, and hence

$$
c_{\mathbf{d}_{1}, \cdots, \mathbf{d}_{s}} r_{\mathbf{d}_{1}} \cdots r_{\mathbf{d}_{s}} \in \mathcal{C}(T) .
$$

Now, by comparing $T$-components of both sides of $(*)$ we get

$$
[N]=\sum c_{\mathbf{d}_{1}, \cdots, \mathbf{d}_{s}} r_{\mathbf{d}_{1}} \cdots r_{\mathbf{d}_{s}}
$$

with each summand $c_{\mathbf{d}_{1}, \cdots, \mathbf{d}_{s}} r_{\mathbf{d}_{1}} \cdots r_{\mathbf{d}_{s}} \in \mathcal{C}(T)$, and hence $[N] \in \mathcal{C}(T)$.

2.9. Proof of Lemma 2.2. Let $T$ be a non-homogeneous tube of rank $m$, and $N$ an indecomposable module of quasi-length $m$ in $T$. Assume that $[N] \in \mathcal{C}(A)$; then by Claim 2.8 we have $[N] \in \mathcal{C}(T)$, and hence $[N] \in \mathcal{C}(T)_{\mathbf{n}}$. Then by 2.7 we know that $[N]$ is a $\mathbb{Q}$-combination of elements in $\Omega$, so $[N]$ can be expressed as

$$
[N]=X_{1}+X_{2}+\cdots+X_{m-1},
$$

where $X_{i}=\sum_{j, L} c_{j, L}\left[E_{j}\right] \cdot[L]$ with $\left[E_{j}\right] \cdot[L] \in \Omega$ and $d(L)=i, 1 \leq i \leq m-1$.

Let $N=E_{1}(m)$ (i.e. the quasi-top and the quasi-length of $N$ are $E_{1}$ and $m$ respectively). Now, we determine $X_{i}$ by induction.

According to (2) in 2.7 we see that every component of $X_{i}$ has the decomposition number $i$ or $i+1$. Note that the left hand side of (5) has a unique component $[N]$, whose decomposition number is 1 . There is only one element in $\Omega$ having the component $[N]$, i.e. $\left[E_{1}\right] \cdot\left[E_{2}(m-1)\right]$. Thus, by comparing the coefficients of the component $[N]$ in both sides of (5), and then by comparing the coefficients of the other components having decomposition number 1 in the both sides of (5), we get

$$
X_{1}=\left[E_{1}\right] \cdot\left[E_{2}(m-1)\right] .
$$

Assume that we have proven the following:

$$
\begin{aligned}
X_{j}= & (-1)^{j-1}\left[E_{j}\right] \cdot\left[E_{1} \oplus \cdots \oplus E_{j-1} \oplus E_{j+1}(m-j)\right] \\
= & (-1)^{j-1}\left[E_{1} \oplus \cdots \oplus E_{j-1} \oplus E_{j}(m-j+1)\right] \\
& +(-1)^{j-1}\left[E_{1} \oplus \cdots \oplus E_{j-1} \oplus E_{j} \oplus E_{j+1}(m-j)\right] .
\end{aligned}
$$

We now derive the term $X_{j+1}$. Note that $X_{1}+\cdots+X_{j-1}+X_{j+2}+\cdots+X_{m-1}$ has no components with decomposition number $j+1$, while by the inductive hypothesis $X_{j}$ has only one component with decomposition number $j+1$, i.e. $\left[E_{1} \oplus \cdots \oplus E_{j-1} \oplus\right.$ $\left.E_{j} \oplus E_{j+1}(m-j)\right]$; also, from 2.7 we see that there is only one element in $\Omega$ of the form $\left[E_{i}\right] \cdot[L]$ with $d(L)=j+1$, which has the component $\left[E_{1} \oplus \cdots \oplus E_{j-1} \oplus E_{j} \oplus\right.$ $\left.E_{j+1}(m-j)\right]$ (this element is $\left.\left[E_{j+1}\right] \cdot\left[E_{1} \oplus \cdots \oplus E_{j} \oplus E_{j+2}(m-j-1)\right]\right)$. Thus, by 
comparing the coefficients of the component $\left[E_{1} \oplus \cdots \oplus E_{j-1} \oplus E_{j} \oplus E_{j+1}(m-j)\right]$ in both sides of $(5)$, we see

$$
X_{j+1}=(-1)^{j}\left[E_{j+1}\right] \cdot\left[E_{1} \oplus \cdots \oplus E_{j} \oplus E_{j+2}(m-j-1)\right]+X
$$

Again by comparing the coefficients of the other components having decomposition number $j+1$ in both sides of (5), we see that $X=0$, i.e.

$$
X_{j+1}=(-1)^{j}\left[E_{j+1}\right] \cdot\left[E_{1} \oplus \cdots \oplus E_{j} \oplus E_{j+2}(m-j-1)\right] .
$$

Thus, by induction we get

$$
\begin{aligned}
{[N]=} & X_{1}+\cdots+X_{m-1} \\
= & \sum_{1 \leq j \leq m-1}(-1)^{j-1}\left[E_{j}\right] \cdot\left[E_{1} \oplus \cdots \oplus E_{j-1} \oplus E_{j+1}(m-j)\right] \\
= & \sum_{1 \leq j \leq m-1}\left((-1)^{j-1}\left[E_{1} \oplus \cdots \oplus E_{j-1} \oplus E_{j}(m-j+1)\right]\right. \\
& \left.\quad+(-1)^{j-1}\left[E_{1} \oplus \cdots \oplus E_{j-1} \oplus E_{j} \oplus E_{j+1}(m-j)\right]\right) \\
= & {[N]+(-1)^{m}\left[E_{1} \oplus \cdots \oplus E_{m}\right], }
\end{aligned}
$$

which is absurd. This completes the proof.

2.10. Proof of Proposition 2.1. Let $M$ be an $A$-module in $T$ with q. $\operatorname{dim} M=$ $(1, \cdots, 1)$. Since $\operatorname{dim} M=\operatorname{dim} E_{1}+\cdots+\operatorname{dim} E_{m}$, it follows from the structure of a tube that $M$ is of the following form:

$$
M=E_{i}\left(v_{1}\right) \oplus E_{i+v_{1}}\left(v_{2}\right) \oplus \cdots \oplus E_{i+v_{1}+\cdots+v_{t-1}}\left(v_{t}\right),
$$

where $v_{1}, \cdots, v_{t} \geq 1 ; v_{1}+v_{2}+\cdots+v_{t}=m$. Set $M_{j}=E_{i+v_{1}+\cdots+v_{j-1}}\left(v_{j}\right), 1 \leq j \leq t$. Then $M=M_{1} \oplus \cdots \oplus M_{t}$. If $t=1$, then $[M] \notin \mathcal{C}(A)$ by Lemma 2.2. If $t \geq 2$, then again by the structure of a tube and the Auslander-Reiten formula we have

$$
\operatorname{Ext}_{A}^{1}\left(M_{j}, M_{j^{\prime}}\right)=\operatorname{Hom}_{A}\left(M_{j^{\prime}}, \tau M_{j}\right)=0 \text { for } j^{\prime} \geq j+2,
$$

and

$$
\operatorname{dim}_{F} \operatorname{Ext}_{A}^{1}\left(M_{j}, M_{j+1}\right)=\operatorname{dim}_{F} \operatorname{Hom}_{A}\left(M_{j+1}, \tau M_{j}\right)=1,
$$

with $F=\operatorname{End}_{A}^{1} M_{j}$ a field (it is $k$ since $A$ is the path algebra). It follows that $\operatorname{dim}_{F} \operatorname{Ext}_{A}^{1}\left(M_{1}, M_{2} \oplus \cdots \oplus M_{t}\right)=1$, and hence by Lemma 2.3 we have

$$
\left[M_{1}\right] \cdot\left[M_{2} \oplus \cdots \oplus M_{t}\right]=c_{1}[M]+c_{2}\left[M^{\prime}\right]
$$

where $c_{1} c_{2} \neq 0$, and $M^{\prime}=E_{i}\left(v_{1}+v_{2}\right) \oplus M_{3} \oplus \cdots \oplus M_{t}$. Since q. $\operatorname{dim} M_{1}$ and q. $\operatorname{dim}\left(M_{2} \oplus \cdots \oplus M_{t}\right)<(1, \cdots, 1)$, it follows from Proposition 2.4 that

$$
\left[M_{1}\right] \cdot\left[M_{2} \oplus \cdots \oplus M_{t}\right] \in \mathcal{C}(A) .
$$

Note that q. $\operatorname{dim} M^{\prime}=(1, \cdots, 1)$ and the number of indecomposable direct summands of $M^{\prime}$ is $t-1$. Now by induction on $t$ we see that $\left[M^{\prime}\right] \notin \mathcal{C}(A)$. This proves that $[M] \notin \mathcal{C}(A)$. 
2.11. An example. We would like to include an example here. Let $A$ be the path algebra of the quiver $\tilde{A_{2}}$, i.e. the quiver with the set of vertices $\{1,2,3\}$ and the set of arrows $\{1 \longrightarrow 2,2 \longrightarrow 3,1 \longrightarrow 3\}$. Then $A$ has only one non-homogeneous tube $T$ with quasi-simple modules $E_{1}$ and $E_{2}$, with $\operatorname{dim} E_{1}=(0,1,0)$ and $\operatorname{dim} E_{2}=$ $(1,0,1)$. Let $N_{1}$ be the indecomposable module in $T$ with quasi-top $E_{1}$ and quasisocle $E_{2}$, and $N_{2}=\tau N_{1}$. Note that $r_{(1,1,1)}=\sum_{H}[H]+\left[N_{1}\right]+\left[N_{2}\right]+\left[E_{1} \oplus E_{2}\right]$, where $H$ ranges over the homogeneous quasi-simple modules with dimension vector $(1,1,1)$. By Proposition 1.1 we know that such an $H$ exists. It is easy to see that $r_{(1,0,0)}=r_{(0,0,1)}=r_{(1,1,0)}=r_{(0,1,1)}=0, \quad r_{(0,1,0)}=\left[E_{1}\right], \quad r_{(1,0,1)}=\left[E_{2}\right]$; and $\left[E_{1}\right] \cdot\left[E_{2}\right]=\left[N_{1}\right]+\left[E_{1} \oplus E_{2}\right], \quad\left[E_{2}\right] \cdot\left[E_{1}\right]=\left[N_{2}\right]+\left[E_{1} \oplus E_{2}\right]$. Thus by Theorem 0.4 we know that $\mathcal{T}_{(1,1,1)}$ is a $\mathbb{Q}$-combinations of $r_{(1,1,1)}, r_{(0,1,0)} \cdot r_{(1,0,1)}$, and $r_{(1,0,1)} \cdot r_{(0,1,0)}$, i.e. we have

$$
\begin{aligned}
\mathcal{T}_{(1,1,1)}=\left\{c_{1} \sum_{H}[H]\right. & +\left(c_{1}+c_{2}\right)\left[N_{1}\right]+\left(c_{1}+c_{3}\right)\left[N_{2}\right] \\
& \left.+\left(c_{1}+c_{2}+c_{3}\right)\left[E_{1} \oplus E_{2}\right] \mid c_{1}, c_{2}, c_{3} \in k\right\} .
\end{aligned}
$$

In particular, we see that $\left[N_{1}\right],\left[N_{2}\right],\left[E_{1} \oplus E_{2}\right] \notin \mathcal{C}(A)$, as Proposition 2.1 claims.

Remark. As we see from the proof, if Claim 2.8 is proved also for non-simply-laced tame hereditary algebras, then Proposition 2.1 is proved for any tame hereditary algebra over any finite field.

\section{INVARIANT SUBSPACES OF SKEW DERIVATIONS}

The aim of this section is to develop machineries for reductions inside the subalgebras $\mathcal{C}(A)$ and $\mathcal{T}$, namely, try to find enough $\mathbb{Q}$-linear transformations $\delta$ of $\mathcal{H}(A)$, such that $\mathcal{C}(A)$ and $\mathcal{T}$ are invariant subspaces.

3.1. Let $A$ be an arbitrary finite-dimensional algebra over a finite field $k$ with $q$ elements. Given an $A$-module $M$, the $\mathbb{Q}$-linear maps $\delta_{M},{ }_{M} \delta$ in $\operatorname{Hom}_{\mathbb{Q}}(\mathcal{H}(A), \mathcal{H}(A))$ are defined in $[\mathrm{CX}]$ as follows:

$$
\delta_{M}([L])=\sum_{[N]} g_{N, M}^{L} \frac{a_{N}}{a_{L}}[N] \quad \text { and } \quad{ }_{M} \delta([L])=\sum_{[N]} g_{M, N}^{L} \frac{a_{N}}{a_{L}}[N] .
$$

If $S(i)$ and $S(j)$ are simple modules, then

$$
\delta_{S(i)}([S(j)])=\frac{\delta_{i j}}{q^{\operatorname{dim}_{k} \operatorname{End}_{A} S(i)}-1}[0]={ }_{S(i)} \delta([S(j)]),
$$

where $\delta_{i j}$ is the Kronecker symbol (here we use the untwisted form of $\delta_{M}$ and ${ }_{M} \delta$ as in [ZZ]). Note that $\delta_{S(i)}$ and ${ }_{S(i)} \delta$ are realizations in $\mathcal{H}(A)$ of Lusztig's derivations $r_{i}$ and ${ }_{i} r$ for $\mathbf{f}$, respectively, see [L3, p.8].

In the rest of this section $A$ is an arbitrary tame hereditary algebra over a field $k$ with $q$ elements (not necessarily a path algebra). For $\mathbf{d} \in \mathbb{N}_{0}^{n}$, recall that in 0.4 the element $r_{\mathbf{d}} \in \mathcal{H}(A)$ is defined as

$$
r_{\mathbf{d}}=\sum_{[N]}[N], \quad \text { where } N \text { ranges over regular modules with } \operatorname{dim} N=\mathbf{d} .
$$

Note that if there is no regular module with dimension vector $\mathbf{d}$, or if $\mathbf{d} \in \mathbb{Z}^{n}-\mathbb{N}_{0}^{n}$, then $r_{\mathbf{d}}:=0$. 
Proposition 3.2. Let $M$ be a regular module, $\mathbf{d} \in \mathbb{N}_{0}^{n}$. Then

$$
\begin{aligned}
\delta_{M}\left(r_{\mathbf{d}}\right) & =\frac{q^{-\langle\mathbf{d}-\operatorname{dim} M, \operatorname{dim} M\rangle}}{a_{M}} r_{\mathbf{d}-\operatorname{dim} M,}, \\
{ }_{M} \delta\left(r_{\mathbf{d}}\right) & =\frac{q^{-\langle\operatorname{dim} M, \mathbf{d}-\operatorname{dim} M\rangle}}{a_{M}} r_{\mathbf{d}-\operatorname{dim} M .} .
\end{aligned}
$$

Proof. By duality we only need to prove (i). By the linearity of $\delta_{M}$ we have

$$
\begin{aligned}
\delta_{M}\left(r_{\mathbf{d}}\right) & =\delta_{M}\left(\sum[N]\right)=\sum \delta_{M}([N])=\sum \sum_{[L]} g_{L, M}^{N} \frac{a_{L}}{a_{N}}[L] \\
& =\sum_{[L]}\left(\sum g_{L, M}^{N} \frac{a_{L}}{a_{N}}\right)[L]=\sum_{[L]} c_{L}[L],
\end{aligned}
$$

where

$$
c_{L}=\sum_{[N]} g_{L, M}^{N} \frac{a_{L}}{a_{N}},
$$

where $[N]$ ranges over all isoclasses of regular modules $N$ with $\operatorname{dim} N=\mathbf{d}$.

Since $c_{L}$ is a sum of non-negative numbers, it follows that $c_{L} \neq 0$ if and only if there exists a regular module $N$ such that $g_{L, M}^{N} \neq 0$. Since $M$ is regular, it follows that $c_{L} \neq 0$ if and only if $L$ is regular with $\operatorname{dim} L=\mathbf{d}-\operatorname{dim} M$.

Now, it remains to prove that if $c_{L} \neq 0$, then

$$
c_{L}=\frac{q^{-\langle\mathbf{d}-\operatorname{dim} M, \operatorname{dim} M\rangle}}{a_{M}} .
$$

For this, recall that the structure constant $g_{L, M}^{N}$ can be expressed as

$$
g_{L, M}^{N}=\frac{a_{N}\left|\operatorname{Ext}_{A}^{1}(L, M)_{N}\right|}{a_{M} a_{L}|\operatorname{Hom}(L, M)|}
$$

where $\operatorname{Ext}_{A}^{1}(L, M)_{N}$ is the set of extension classes in $\operatorname{Ext}_{A}^{1}(L, M)$ with middle term $N$ (see [GP] and [Rm]; also [G1] and [R11]).

Indeed, if $L$ is regular with $\operatorname{dim} L=\mathbf{d}-\operatorname{dim} M$, then we have

$$
\begin{aligned}
c_{L} & =\sum g_{L, M}^{N} \frac{a_{L}}{a_{N}}=\sum \frac{a_{N}\left|\operatorname{Ext}_{A}^{1}(L, M)_{N}\right|}{a_{M} a_{L}|\operatorname{Hom}(L, M)|} \frac{a_{L}}{a_{N}} \\
& =\frac{1}{a_{M}|\operatorname{Hom}(L, M)|} \sum_{[N]}\left|\operatorname{Ext}_{A}^{1}(L, M)_{N}\right| \\
& =\frac{\left|\operatorname{Ext}_{A}^{1}(L, M)\right|}{a_{M}|\operatorname{Hom}(L, M)|}=\frac{q^{-\langle\operatorname{dim} L, \operatorname{dim} M\rangle}}{a_{M}}=\frac{q^{-\langle\mathbf{d}-\operatorname{dim} M, \operatorname{dim} M\rangle}}{a_{M}} .
\end{aligned}
$$

Proposition 3.3. Let $S$ be a quasi-simple regular module, $M$ and $N$ regular modules. Then

$$
\begin{aligned}
& \delta_{S}([M] \cdot[N])=\delta_{S}([M]) \cdot[N]+q^{-\langle M, S\rangle}[M] \cdot \delta_{S}([N]), \\
& { }_{S} \delta([M] \cdot[N])=q^{-\langle S, N\rangle}{ }_{S} \delta([M]) \cdot[N]+[M] \cdot{ }_{S} \delta([N]) .
\end{aligned}
$$

Remark. If $S$ is a simple $A$-module, then Proposition 3.3 is true for two arbitrary modules $M$ and $N$; this is proved in [CX] (also [ZZ, 4.1] for the untwisted case). Note that if $S$ is not quasi-simple, then the above formulas are not true. 
Proof. By duality we only need to prove (i). By the definitions we have

$$
\begin{gathered}
\delta_{S}([M] \cdot[N])=\delta_{S}\left(\sum_{[L]} g_{M, N}^{L}[L]\right)=\sum_{[L],[V]} g_{M, N}^{L} g_{V, S}^{L} \frac{a_{V}}{a_{L}}[V] ; \\
\delta_{S}([M]) \cdot[N]=\left(\sum_{\left[V^{\prime}\right]} g_{V^{\prime}, S}^{M} \frac{a_{V^{\prime}}}{a_{M}}\left[V^{\prime}\right]\right) \cdot[N]=\sum_{\left[V^{\prime}\right],[V]} g_{V^{\prime}, S}^{M} g_{V^{\prime}, N}^{V} \frac{a_{V^{\prime}}}{a_{M}}[V] ; \\
{[M] \cdot \delta_{S}([N])=[M] \cdot\left(\sum_{[U]} g_{U, S}^{N} \frac{a_{U}}{a_{N}}[U]\right)=\sum_{[U],[V]} g_{U, S}^{N} g_{M, U}^{V} \frac{a_{U}}{a_{N}}[V] .}
\end{gathered}
$$

So, in order to prove (i), it will suffice to prove that the following equality holds for any module $V$ :

$$
\sum_{[L]} g_{M, N}^{L} g_{V, S}^{L} \frac{a_{V}}{a_{L}}=\sum_{\left[V^{\prime}\right]} g_{V^{\prime}, S}^{M} g_{V^{\prime}, N}^{V} \frac{a_{V^{\prime}}}{a_{M}}+q^{-\langle M, S\rangle} \sum_{[U]} g_{U, S}^{N} g_{M, U}^{V} \frac{a_{U}}{a_{N}} .
$$

In fact, the equality $(*)$ is essentially a special situation of Green's formula in G1. Theorem 2]. In order to see this, let

$$
N_{1}(M, N, V, S)=\sum_{[L]} g_{M, N}^{L} g_{V, S}^{L} \frac{a_{M} a_{N} a_{V} a_{S}}{a_{L}}
$$

and

$$
N_{2}(M, N, V, S)=\sum_{[X],[Y],\left[X^{\prime}\right],\left[Y^{\prime}\right]} q^{-\left\langle X, Y^{\prime}\right\rangle} g_{X, X^{\prime}}^{M} g_{Y, Y^{\prime}}^{N} g_{X, Y}^{V} g_{X^{\prime}, Y^{\prime}}^{S} a_{X} a_{Y} a_{X^{\prime}} a_{Y^{\prime}} .
$$

Let $g_{X, X^{\prime}}^{M} g_{Y, Y^{\prime}}^{N} g_{X, Y}^{V} g_{X^{\prime}, Y^{\prime}}^{S} \neq 0$. Since $M$ is regular, it follows that $X^{\prime}$ has no preinjective summands, and hence the defect $\partial\left(X^{\prime}\right) \leq 0$. Similarly, we have $\partial\left(Y^{\prime}\right) \leq$ 0 . However, $g_{X^{\prime}, Y^{\prime}}^{S} \neq 0$ means that $\partial\left(X^{\prime}\right)+\partial\left(Y^{\prime}\right)=\partial(S)=0$; this forces both $X^{\prime}$ and $Y^{\prime}$ to be regular. Since $S$ is a quasi-simple regular module, it follows that $g_{X^{\prime}, Y^{\prime}}^{S} \neq 0$ if and only if one of $X^{\prime}, Y^{\prime}$ is $S$ and the other is 0 , and hence we have

$$
N_{2}(M, N, V, S)=\sum_{[X]} g_{X, S}^{M} g_{X, N}^{V} a_{X} a_{N} a_{S}+\sum_{[Y]} q^{-\langle M, S\rangle} g_{Y, S}^{N} g_{M, Y}^{V} a_{M} a_{Y} a_{S} .
$$

Now, by Green's formula in G1, Theorem 2] we have

$$
\frac{1}{a_{M} a_{N} a_{S}} N_{1}(M, N, V, S)=\frac{1}{a_{M} a_{N} a_{S}} N_{2}(M, N, V, S),
$$

which is exactly the desired equality $(*)$.

Corollary 3.4. If $S$ is a quasi-simple regular module and $M_{1}, \cdots, M_{t}(t \geq 2)$ are regular modules, or if $S$ is a simple module and $M_{1}, \cdots, M_{t}$ are arbitrary modules, then we have

$$
\begin{aligned}
& \delta_{S}\left(\left[M_{1}\right] \cdots\left[M_{t}\right]\right) \\
& \quad=\sum_{0 \leq i \leq t-1} q^{-\left\langle M_{1} \oplus \cdots \oplus M_{i}, S\right\rangle}\left[M_{1}\right] \cdots\left[M_{i}\right] \cdot \delta_{S}\left(\left[M_{i+1}\right]\right) \cdot\left[M_{i+2}\right] \cdots\left[M_{t}\right], \\
& \quad \begin{array}{l}
\delta \delta\left(\left[M_{1}\right]\right. \\
\quad=\sum_{0 \leq i \leq t-1} q^{-\left\langle S, M_{i+2} \oplus \cdots \oplus M_{t}\right\rangle}\left[M_{1}\right] \cdots\left[M_{i}\right] \cdot{ }_{S} \delta\left(\left[M_{i+1}\right]\right) \cdot\left[M_{i+2}\right] \cdots\left[M_{t}\right] .
\end{array}
\end{aligned}
$$


Proof. The formulae follow from Proposition 3.3 and its remark by induction. We omit the details.

Recall that the subalgebra $\mathcal{T}$ of $\mathcal{C}(A)$ is generated by all elements $r_{\mathbf{d}}$, where $\mathbf{d}$ runs over $\mathbb{N}_{0}^{n}$. Thus, $\mathcal{T}$ is a $\mathbb{Q}$-span of the elements of the form $r_{\mathbf{d}_{1}} \cdots r_{\mathbf{d}_{t}}$, with $\mathbf{d}_{1}, \cdots, \mathbf{d}_{t} \in \mathbb{N}_{0}^{n}$ and $t \in \mathbb{N}_{0}$ (note that $r_{0}=[0]$, the identity of $\mathcal{T}$ ).

Theorem 3.5. Let $S$ be a quasi-simple regular module. Then $\delta_{S},{ }_{S} \delta \in \operatorname{Hom}_{\mathbb{Q}}(\mathcal{T}, \mathcal{T})$. More precisely, let $\mathbf{d}_{1}, \cdots, \mathbf{d}_{t} \in \mathbb{N}_{0}^{n}$. Then we have

$$
\begin{aligned}
& \delta_{S}\left(r_{\mathbf{d}_{1}} \cdots r_{\mathbf{d}_{t}}\right) \\
& =\frac{1}{a_{S}} \sum_{0 \leq i \leq t-1} q^{-\left\langle\mathbf{d}_{1}+\cdots+\mathbf{d}_{i+1}-\operatorname{dim} S, \operatorname{dim} S\right\rangle} r_{\mathbf{d}_{1}} \cdots r_{\mathbf{d}_{i}} \cdot r_{\mathbf{d}_{i+1}-\operatorname{dim} S} r_{\mathbf{d}_{i+2}} \cdots r_{\mathbf{d}_{t}},
\end{aligned}
$$

and

$$
\begin{aligned}
& { }_{S} \delta\left(r_{\mathbf{d}_{1}} \cdots r_{\mathbf{d}_{t}}\right) \\
& =\frac{1}{a_{S}} \sum_{0 \leq i \leq t-1} q^{-\left\langle\operatorname{dim} S, \mathbf{d}_{i+1}+\cdots+\mathbf{d}_{t}-\operatorname{dim} S\right\rangle} r_{\mathbf{d}_{1}} \cdots r_{\mathbf{d}_{i}} \cdot r_{\mathbf{d}_{i+1}-\operatorname{dim} S} r_{\mathbf{d}_{i+2}} \cdots r_{\mathbf{d}_{t}} .
\end{aligned}
$$

Remark. For later applications, we emphasize that the quasi-simple regular module $S$ in Theorem 3.5 need not be a stone; it can be a homogeneous quasi-simple.

Proof. By the definition, Corollary 3.4 and Proposition 3.2 we have

$$
\begin{aligned}
& \delta_{S}\left(r_{\mathbf{d}_{1}} \cdots r_{\mathbf{d}_{t}}\right) \\
& =\delta_{S}\left(\sum_{\left[N_{1}\right]}\left[N_{1}\right] \cdots \sum_{\left[N_{t}\right]}\left[N_{t}\right]\right) \\
& =\delta_{S}\left(\sum_{\left[N_{1}\right], \cdots,\left[N_{t}\right]}\left[N_{1}\right] \cdots\left[N_{t}\right]\right) \\
& =\sum_{\left[N_{1}\right], \cdots,\left[N_{t}\right]} \delta_{S}\left(\left[N_{1}\right] \cdots\left[N_{t}\right]\right) \\
& =\sum_{\left[N_{1}\right], \cdots,\left[N_{t}\right] 0 \leq i \leq t-1} \sum^{-\left\langle\mathbf{d}_{1}+\cdots+\mathbf{d}_{i}, \operatorname{dim} S\right\rangle}\left[N_{1}\right] \cdots\left[N_{i}\right] \cdot \delta_{S}\left(\left[N_{i+1}\right]\right) \cdot\left[N_{i+2}\right] \cdots\left[N_{t}\right] \\
& =\sum_{0 \leq i \leq t-1} q^{-\left\langle\mathbf{d}_{1}+\cdots+\mathbf{d}_{i}, \operatorname{dim} S\right\rangle} \sum_{\left[N_{1}\right], \cdots,\left[N_{t}\right]}\left[N_{1}\right] \cdots\left[N_{i}\right] \cdot \delta_{S}\left(\left[N_{i+1}\right]\right) \cdot\left[N_{i+2}\right] \cdots\left[N_{t}\right] \\
& =\sum_{0 \leq i \leq t-1} q^{-\left\langle\mathbf{d}_{1}+\cdots+\mathbf{d}_{i}, \operatorname{dim} S\right\rangle} \prod_{1 \leq j \leq i}\left(\sum_{\left[N_{j}\right]}\left[N_{j}\right]\right) \cdot \delta_{S}\left(\sum_{\left[N_{i+1}\right]}\left[N_{i+1}\right]\right) \cdot \prod_{i+2 \leq j \leq t}\left(\sum_{\left[N_{j}\right]}\left[N_{j}\right]\right) \\
& =\sum_{0 \leq i \leq t-1} q^{-\left\langle\mathbf{d}_{1}+\cdots+\mathbf{d}_{i}, \operatorname{dim} S\right\rangle} r_{\mathbf{d}_{1}} \cdots r_{\mathbf{d}_{i}} \cdot \delta_{S}\left(r_{\mathbf{d}_{i+1}}\right) \cdot r_{\mathbf{d}_{i+2}} \cdots r_{\mathbf{d}_{t}} \\
& =\frac{1}{a_{S}} \sum_{0 \leq i \leq t-1} q^{-\left\langle\mathbf{d}_{1}+\cdots+\mathbf{d}_{i+1}-\operatorname{dim} S, \operatorname{dim} S\right\rangle} r_{\mathbf{d}_{1}} \cdots r_{\mathbf{d}_{i}} \cdot r_{\mathbf{d}_{i+1}-\operatorname{dim} S} \cdot r_{\mathbf{d}_{i+2}} \cdots r_{\mathbf{d}_{t}} .
\end{aligned}
$$

Dually, the other formula can be proved.

3.6. Let $A$ be an arbitrary finite-dimensional algebra over a finite field. Consider the $\mathbb{Q}$-linear maps $\phi_{1}, \phi_{2}: \mathcal{H}(A) \longrightarrow \operatorname{Hom}_{\mathbb{Q}}(\mathcal{H}(A), \mathcal{H}(A))$ given by $\phi_{1}([M])=\delta_{M}$, $\phi_{2}([M])={ }_{M} \delta$. 
Lemma $([\mathrm{CX}])$. (i) $\phi_{1}$ is a $\mathbb{Q}$-algebra homomorphism, i.e. $\phi_{1}([M] \cdot[N])=\delta_{M} \cdot \delta_{N}$.

(ii) $\phi_{2}$ is a $\mathbb{Q}$-algebra anti-homomorphism, i.e. $\phi_{2}([M] \cdot[N])={ }_{N} \delta \cdot{ }_{M} \delta$.

In particular, let $M_{1}, \cdots, M_{m}$ be arbitrary $A$-modules with $\operatorname{Ext}_{A}^{1}\left(M_{i}, M_{j}\right)=0$ for $i<j$. Then we have

$$
\delta_{M_{1} \oplus \cdots \oplus M_{m}}=c \delta_{M_{1}} \cdots \delta_{M_{m}}
$$

and

$$
M_{1} \oplus \cdots \oplus M_{m} \delta=c_{M_{m}} \delta \cdots_{M_{1}} \delta
$$

where

$$
c=\frac{\left[M_{1} \oplus \cdots \oplus M_{m}\right]}{\left[M_{1}\right] \cdots\left[M_{m}\right]}
$$

is a positive number.

Theorem $3.7([\mathrm{ZZ}])$. If $[M] \in \mathcal{C}(A)$, then $\delta_{M},{ }_{M} \delta \in \operatorname{Hom}_{\mathbb{Q}}(\mathcal{C}(A), \mathcal{C}(A))$.

Proof. For completeness we include the proof here. By duality we only need to prove $\delta_{M} \in \operatorname{Hom}_{\mathbb{Q}}(\mathcal{C}(A), \mathcal{C}(A))$.

Assume that $[M]=\sum b_{i_{1}, \cdots, i_{t}}\left[S\left(i_{1}\right)\right] \cdots\left[S\left(i_{t}\right)\right]$, where the $S\left(i_{j}\right)$ 's are simple $A$ modules. Then by Lemma 3.6 we have

$$
\delta_{M}=\phi_{1}([M])=\sum b_{i_{1}, \cdots, i_{t}} \delta_{S\left(i_{1}\right)} \cdots \delta_{S\left(i_{t}\right)} .
$$

For any element $X \in \mathcal{C}(A)$, say, $X=\sum c_{j_{1}, \cdots, j_{s}}\left[S\left(j_{1}\right)\right] \cdots\left[S\left(j_{s}\right)\right]$, according to Corollary 3.4 and the formula (6) we see that

$$
\delta_{M}(X)=\sum b_{i_{1}, \cdots, i_{t}} c_{j_{1}, \cdots, j_{s}} \delta_{S\left(i_{1}\right)} \cdots \delta_{S\left(i_{t}\right)}\left(\left[S\left(j_{1}\right)\right] \cdots\left[S\left(j_{s}\right)\right]\right) \in \mathcal{C}(A) .
$$

This completes the proof.

Corollary 3.8. Let $M$ be a regular $A$-module with $[M] \in \mathcal{C}(A)$. Then $\delta_{M},{ }_{M} \delta \in$ $\operatorname{Hom}_{\mathbb{Q}}(\mathcal{T}, \mathcal{T})$.

Proof. It follows from Theorem 3.7 that $\delta_{M}(\mathcal{T}) \subseteq \mathcal{C}(A)$ and ${ }_{M} \delta(\mathcal{T}) \subseteq \mathcal{C}(A)$. Since $M$ is regular by assumption, and $\mathcal{C}(A)=\mathcal{P} \cdot \mathcal{T} \cdot \mathcal{I}$ by Theorem 0.4 , it follows that $\delta_{M}(\mathcal{T}) \subseteq \mathcal{T}$, and ${ }_{M} \delta(\mathcal{T}) \subseteq \mathcal{T}$

Corollary 3.9. (i) Let $E=E_{1} \oplus \cdots \oplus E_{t}$ with all $E_{i}$ 's quasi-simple regular modules, such that if $t \geq 2$ then $\operatorname{Ext}_{A}^{1}\left(E_{j}, E_{i}\right)=0$ for $j>i$. Then we have $\delta_{M},{ }_{M} \delta \in$ $\operatorname{Hom}_{\mathbb{Q}}(\mathcal{T}, \mathcal{T})$.

(ii) Let $\left(M_{1}, \cdots, M_{m}\right)$ be an exceptional sequence (i.e. all $M_{i}$ 's are stones with $\operatorname{Hom}_{A}\left(M_{j}, M_{i}\right)=0=\operatorname{Ext}_{A}^{1}\left(M_{j}, M_{i}\right)$ for $\left.j \geq i\right)$. Let $M=M_{1} \oplus \cdots \oplus M_{m}$. Then we have $\delta_{M},{ }_{M} \delta \in \operatorname{Hom}_{\mathbb{Q}}(\mathcal{C}(A), \mathcal{C}(A))$. In particular, if $M$ is in addition a regular module, then $\delta_{M},{ }_{M} \delta \in \operatorname{Hom}_{\mathbb{Q}}(\mathcal{T}, \mathcal{T})$.

Proof. The assertion (i) is a direct consequence of Lemma 3.6 and Theorem 3.5. For (ii), note that $[M] \in \mathcal{C}(A)$ by [Z1, Theorem 2], and hence the assertion follows from Theorem 3.7 and Corollary 3.8.

Remark 3.10. Corollary 3.8 does not imply Theorem 3.5, since by Lemma 5.2 below we know that $[S] \notin \mathcal{C}(A)$ for a homogeneous quasi-simple module $S$. 


\section{Modules With SINCERE QUASI-DIMENSION VECTOR}

Let $k$ be a finite field with $|k| \neq 2$. The aim of this section is to prove the following statement.

Proposition 4.1. Assume that $A$ is the $k$-path algebra of a Euclidean quiver. Let $T$ be a non-homogeneous tube and $M$ an arbitrary $A$-module with $M \in T$. Then $[M] \in \mathcal{C}(A)$ if and only if $\mathbf{q} \cdot \operatorname{dim} M \nsupseteq(1, \cdots, 1)$, i.e. q. $\operatorname{dim} M$ is non-sincere.

4.2. The central ideal of the proof is to try to find suitable modules $E$ with $[E] \in \mathcal{T}$, such that after applying Theorem 3.7 finitely many times we can reduce $M$ to the case of $\mathbf{q} \cdot \operatorname{dim} M=(1, \cdots, 1)$, for which Proposition 2.1 can be applied.

In the following subsections 4.3-4.7 let $A$ be an arbitrary finite-dimensional tame hereditary algebra over any finite field (not necessarily a path algebra, no restrictions on $|k|)$, and let $E_{1}, \cdots, E_{m}$ be the quasi-simple modules in $T$ such that $\tau E_{1}=E_{i+1}, 1 \leq i \leq m-1$, and $\tau E_{m}=E_{1}$. Let $M$ be an $A$-module in $T$ with q. $\operatorname{dim} M=\left(d_{1}, \cdots, d_{m}\right)>(1, \cdots, 1)$. Denote by $s_{i}$ the number of indecomposable direct summands of $M$ with quasi-socle $E_{i}$, and denote by $t_{i}$ the number of indecomposable direct summands of $M$ with quasi-top $E_{i}$. Then we have $0 \leq s_{i}, t_{i}, \leq d_{i}$ for $1 \leq i \leq m$. We keep this notations of $T, m, E_{i}$ 's, $d_{i}$ 's, $s_{i}$ 's and $t_{i}$ 's throughout this section. Note that $s_{i}$ is exactly the number of indecomposable direct summands $M_{j}$ of $M$ with $\operatorname{Hom}_{A}\left(E_{i}, M_{j}\right) \neq 0$, and that $t_{i}$ is exactly the number of indecomposable direct summands $M_{j}$ of $M$ with $\operatorname{Hom}_{A}\left(M_{j}, E_{i}\right) \neq 0$.

Lemma 4.3. Let $M$ be an $A$-module in $T$ with $\mathbf{q} \cdot \operatorname{dim} M>(1, \cdots, 1)$.

(i) If there exists an index $i$ such that $2 \leq s_{i}+1 \leq d_{i}$, then there exist a module $E \in T$ with $[E] \in \mathcal{C}(A)$, and a non-zero number $c$, such that $\delta_{E}([M])=c[N]$ with $N \in T$ and $(1, \cdots, 1) \leq \mathbf{q} \cdot \operatorname{dim} N<\mathbf{q} \cdot \operatorname{dim} M$.

(ii) If there exists an index isuch that $2 \leq t_{i}+1 \leq d_{i}$, then there exist a module $E \in T$ with $[E] \in \mathcal{C}(A)$, and a non-zero number $c$, such that ${ }_{E} \delta([M])=c[N]$ with $N \in T$ and $(1, \cdots, 1) \leq \mathbf{q} \cdot \operatorname{dim} N<\mathbf{q} \cdot \operatorname{dim} M$.

Proof. By duality it suffices to prove (i). Let $E=E_{i}^{s_{i}}$. Since $E_{i}$ is a stone, it follows from [Z1 Theorem 2] that $[E] \in \mathcal{C}(A)$. Write $M$ as $M=U \oplus V$, where $U$ is exactly the direct sum of all indecomposable direct summands of $M$ with quasi-socle $E_{i}$. Then $\operatorname{Hom}_{A}\left(E_{i}, V\right)=0$, and $E$ is exactly the quasi-socle of $U$. It follows that $U$ has only one submodule isomorphic to $E$. Let $0 \longrightarrow E \longrightarrow M \longrightarrow N \longrightarrow 0$ be an arbitrary short exact sequence. Then $N \simeq V \oplus(U / E)$. It follows that

$$
\delta_{E}([M])=g_{V \oplus(U / E), E}^{M} \frac{a_{V \oplus(U / E)}}{a_{M}}[V \oplus(U / E)]=\frac{a_{V \oplus(U / E)}}{a_{M}}[V \oplus(U / E)] .
$$

Note that $(1, \cdots, 1) \leq \mathbf{q} \cdot \operatorname{dim}(V \oplus(U / E))<\mathbf{q} \cdot \operatorname{dim} M$. This completes the proof.

For $x=\left(x_{1}, \cdots, x_{m}\right) \in \mathbb{N}_{0}^{m}$, the set $\left\{i \mid 1 \leq i \leq m, x_{i} \neq 0\right\}$ is called the support of $x$.

Lemma 4.4. Let $M$ be an $A$-module in $T$ with q. $\operatorname{dim} M>(1, \cdots, 1)$. Assume that $M$ has no indecomposable direct summands of quasi-length $m$. Then at least one of the following two statements holds:

(i) There exist a module $E \in T$ with $[E] \in \mathcal{C}(A)$, and a non-zero number $c$, such that $\delta_{E}([M])=c[N]$ with $N \in T$ and $(1, \cdots, 1) \leq \mathbf{q} \cdot \operatorname{dim} N<\mathbf{q} \cdot \operatorname{dim} M$.

(ii) There exist a module $E \in T$ with $[E] \in \mathcal{C}(A)$, and a non-zero number $c$, such that ${ }_{E} \delta([M])=c[N]$ with $N \in T$ and $(1, \cdots, 1) \leq \mathbf{q} \cdot \operatorname{dim} N<\mathbf{q} \cdot \operatorname{dim} M$. 
Proof. By Lemma 4.3 it suffices to prove that there exists an index $i$ such that $2 \leq s_{i}+1 \leq d_{i}$, or, that there exists an index $i$ such that $2 \leq t_{i}+1 \leq d_{i}$.

Assume that this is not true, i.e. for every index $i$ with $s_{i} \neq 0$ we have $s_{i}=d_{i}$, and for every index $j$ with $t_{j} \neq 0$ we have $t_{j}=d_{j}$.

Let $M=M_{1} \oplus \cdots \oplus M_{t}$ with all $M_{j}$ indecomposable. Denote by $U_{i}$ the direct sum of all $M_{j}$ with quasi-socle $E_{i}$. Note that $M=U_{1} \oplus \cdots \oplus U_{m}$, and some $U_{i}$ may be zero.

Note that the quasi-length of $M_{j}, 1 \leq j \leq t$, is smaller than $m$. Otherwise, we see that the quasi-length of $M_{j}$ is bigger than $m$, since by assumption the quasilength of $M_{j}$ is not $m$. Then we get $d_{i}>s_{i}$, where $E_{i}$ is the quasi-socle of $M_{j}$, this contradicts the assumption.

Since all (non-zero) indecomposable direct summands of $U_{i}$ have same quasi-socle $E_{i}$, it follows that the support of $U_{i}$ coincides with the support of the indecomposable direct summand $M_{i_{j}}$ of $U_{i}$ which is of biggest quasi-length.

Note that the $i$-th component of q. $\operatorname{dim} U_{i}$ is exactly $s_{i}=d_{i}$. Since by assumption for every index $i$ with $s_{i} \neq 0$ we have $d_{i}=s_{i}$, it follows that for $i \neq j$ the support of $\mathbf{q} \cdot \operatorname{dim} U_{i}$ and the support of $\mathbf{q} \cdot \operatorname{dim} U_{j}$ are disjoint. But q. $\operatorname{dim} U=$ q. $\operatorname{dim} U_{1}+\cdots+$ q. $\operatorname{dim} U_{m}>(1, \cdots, 1)$, it follows that there exists an index $i$ such that $s_{i} \geq 2$. For every index $i$ such that $s_{i} \geq 2, U_{i}$ has to be $s_{i}$ copies of an indecomposable stone, since otherwise we get $d_{j}>t_{j}>0$ for some $j$.

Now let $s_{i} \geq 2$ and $U_{i}=X^{s_{i}}$ with $X$ indecomposable. Set $E=X^{s_{i}-1}$. Then $[E] \in \mathcal{C}(A)$. Note that $\operatorname{Hom}_{A}\left(E, U_{j}\right)=0$ for $j \neq i$. It follows that $\delta_{E}([M])=$ $c\left[X \oplus\left(\bigoplus_{j \neq i} U_{j}\right)\right]$ for a non-zero number $c$, with

$$
(1, \ldots, 1) \leq \mathbf{q} \cdot \operatorname{dim}\left(X \oplus\left(\bigoplus_{j \neq i} U_{j}\right)\right)<\mathbf{q} \cdot \operatorname{dim} M .
$$

This completes the proof.

Lemma 4.5. Let $M=H^{t} \oplus U$ be a module in $T$ with $U$ non-zero, where $t$ is a positive integer, $H$ is an indecomposable with quasi-length $m$, and $H$ is not a direct summand of $U$. Then at least one of the following two statements holds:

(i) There exist a module $E \in T$ with $[E] \in \mathcal{C}(A)$, and a non-zero number $c$, such that $\delta_{E}([M])=c[N]$ with $N \in T$ and $(1, \cdots, 1) \leq$ q. $\operatorname{dim} N<$ q. $\operatorname{dim} M$.

(ii) There exist a module $E \in T$ with $[E] \in \mathcal{C}(A)$, and a non-zero number $c$, such that ${ }_{E} \delta([M])=c[N]$ with $N \in T$ and $(1, \cdots, 1) \leq$ q. $\operatorname{dim} N<$ q. $\operatorname{dim} M$.

Proof. By Lemma 4.3 it suffice to prove that the following two situations cannot occur simultaneously.

(I) For every index $i$ with $s_{i} \neq 0$ we have $d_{i}=s_{i}$.

(II) For every index $i$ with $t_{i} \neq 0$ we have $d_{i}=t_{i}$.

Assume that both (I) and (II) are satisfied. Without loss of generality, let $E_{1}$ be the quasi-socle of $H$.

First, we claim that every indecomposable direct summand of $U$ also has the quasi-socle $E_{1}$.

(Otherwise, let $E_{i}(i \neq 1)$ be the quasi-socle of an indecomposable direct summand $V$ of $U$. Then $d_{i}>s_{i}$, which contradicts the assumption (I).)

Secondly, since all indecomposable direct summands of $U$ have the same quasisocle $E_{1}$, it follows that the support of $U$ coincides with the support of the indecomposable direct summand $V$ of $U$ which is of biggest quasi-length. Thus, the 
quasi-length of $V$ is smaller than $m$. In fact, since $V$ and $H$ have the same quasisocle $E_{1}$ and by assumption $H$ is not a direct summand of $U$, it follows that the quasi-length of $V$ is not $m$; and also the quasi-length of $V$ cannot bigger than $m$, since $d_{1}=s_{1}$ by the assumption (I).

Finally, let $E_{i}$ be the quasi-top of an indecomposable direct summand $V$ of $U$. Then $i \neq m$, since the quasi-socle of $V$ is $E_{1}$ and the quasi-length of $V$ is smaller than $m$. In this way we see that $t_{i}<d_{i}$, which contradicts the assumption (II). This completes the proof.

Lemma 4.6. Let $M=H^{t}$ with $t \geq 2$ and $H$ an indecomposable in $T$ of quasilength $m$. Then

(i) There exist a module $E \in T$ with $[E] \in \mathcal{C}(A)$, and a non-zero number $c$, such that $\delta_{E}([M])=c[N]$ with $N \in T$ and $(1, \cdots, 1) \leq \mathbf{q} \cdot \operatorname{dim} N<\mathbf{q} \cdot \operatorname{dim} M$.

(ii) There exist a module $E \in T$ with $[E] \in \mathcal{C}(A)$, and a non-zero number $c$, such that ${ }_{E} \delta([M])=c[N]$ with $N \in T$ and $(1, \cdots, 1) \leq \mathbf{q} \cdot \operatorname{dim} N<\mathbf{q} \cdot \operatorname{dim} M$.

Proof. By duality it suffices to prove (i). Assume that $E_{1}$ is the quasi-socle of $H$. Let $E=E_{1}^{t-1}$. Since $E_{1}$ is a stone, it follows from [Z1. Theorem 2] that $[E] \in \mathcal{C}(A)$. Let $0 \longrightarrow E \longrightarrow M \longrightarrow N \longrightarrow 0$ be an arbitrary short exact sequence. Then $N$ has to be the module $H \oplus U^{t-1}$, where $U$ is the indecomposable in $T$ with quasi-length $m-1$ and quasi-top $E_{m}$. It follows that $\delta_{E}([M])=c\left[H \oplus U^{t-1}\right]$, with $(1, \cdots, 1)<\mathbf{q} \cdot \operatorname{dim}\left(H \oplus U^{t-1}\right)=(1, t, \cdots, t)<\mathbf{q} \cdot \operatorname{dim} M$. This completes the proof.

By Lemmas 4.4-4.6 we get the following

Corollary 4.7. Let $T$ be a non-homogeneous tube of $A$, and let $M$ be an $A$-module in $T$ with $\mathbf{q} . \operatorname{dim} M>(1, \cdots, 1)$. Then at least one of the following two statements holds

(i) There exist a module $E \in T$ with $[E] \in \mathcal{C}(A)$, and a non-zero number $c$, such that $\delta_{E}([M])=c[N]$ with $N \in T$ and $(1, \cdots, 1) \leq \mathbf{q} \cdot \operatorname{dim} N<\mathbf{q} \cdot \operatorname{dim} M$.

(ii) There exist a module $E \in T$ with $[E] \in \mathcal{C}(A)$, and a non-zero number $c$, such that ${ }_{E} \delta([M])=c[N]$ with $N \in T$ and $(1, \cdots, 1) \leq \mathbf{q} \cdot \operatorname{dim} N<\mathbf{q} \cdot \operatorname{dim} M$.

4.8. Proof of Proposition 4.1. The sufficiency follows from Proposition 2.4.

Conversely, let $[M] \in \mathcal{C}(A)$. Then by Proposition 2.1 we know that q. $\operatorname{dim} M \neq$ $(1, \cdots, 1)$. We claim that q. $\operatorname{dim} M$ also cannot be bigger than $(1, \cdots, 1)$.

Otherwise, if q. $\operatorname{dim} M>(1, \cdots, 1)$, then by Corollary 4.7 there exist a module $E \in T$ with $[E] \in \mathcal{C}(A)$, and a non-zero number $c$, such that $\delta_{E}([M])=c[N]$, or ${ }_{E} \delta([M])=c[N]$, where $N \in T$ and $(1, \cdots, 1) \leq \mathbf{q} \cdot \operatorname{dim} N<\mathbf{q} \cdot \operatorname{dim} M$. Now, by Theorem 3.7, $[N] \in \mathcal{C}(A)$. Then again by Proposition 2.1 we see that $\mathbf{q} . \operatorname{dim} N>$ $(1, \cdots, 1)$. In this way we get a contradiction, since this process can be repeated infinitely! This completes the proof.

\section{Modules in Homogeneous tubes}

In this section we assume that $|k| \geq 4$, and $A$ is the path algebra of a Euclidean quiver. Our aim is to prove the following proposition, which is crucial to the proof of the main result.

Proposition 5.1. Let $T$ be a homogeneous tube, and let $M$ be an arbitrary nonzero A-module with $M \in T$. Then $[M] \notin \mathcal{C}(A)$. 
In order to prove Proposition 5.1, we first prove the following special case of $M$ indecomposable, which has been proved in [ZZ §3] (however, the condition $|k| \geq 4$ was really used but not indicated there, and also Proposition 1.4 was used without a proof there). In order to indicate where Proposition 1.4 is used, and for completeness, we include the proof of Lemma 5.2 here.

Lemma 5.2. Let $N$ be a non-zero indecomposable A-module in a homogeneous tube. Then $[N] \notin \mathcal{C}(A)$.

Proof. Let $N \in T$ with $T$ a homogeneous tube. Assume that $[N] \in \mathcal{C}(A)$. Then by Theorem 0.4 we have $[N] \in \mathcal{T}$. Thus, we have the following expression:

$$
[N]=\sum c_{\mathbf{d}_{1}, \cdots, \mathbf{d}_{m}} r_{\mathbf{d}_{1}} \cdots r_{\mathbf{d}_{m}}
$$

where $c_{\mathbf{d}_{1}, \cdots, \mathbf{d}_{m}} r_{\mathbf{d}_{1}} \cdots r_{\mathbf{d}_{m}} \neq 0, \mathbf{d}_{1}, \cdots, \mathbf{d}_{m} \in \mathbb{N}_{0}^{n}-\{0\}$, and $\mathbf{d}_{1}+\cdots+\mathbf{d}_{m}=\operatorname{dim} N$.

Let $\lambda \mathbf{n}$ be the dimension vector of the quasi-simple module in $T$, where $\lambda$ is a positive integer. By Proposition 1.4 we can choose another homogeneous tube $T^{\prime}$ different from $T$ such that the dimension vector of the quasi-simple module in $T^{\prime}$ is also $\lambda \mathbf{n}$. Let $N^{\prime}$ be the indecomposable in $T^{\prime}$ with $\operatorname{dim} N^{\prime}=\operatorname{dim} N$. Now, denote by $\Theta_{T}$ the set of the elements $\left(\mathbf{d}_{1}, \cdots, \mathbf{d}_{m}\right)$ such that the product $r_{\mathbf{d}_{1}} \cdots r_{\mathbf{d}_{m}}$ in the above sum has the component $[N]$ (see 2.5 Notation). Similarly, denote by $\Theta_{T^{\prime}}$ the set of the elements $\left(\mathbf{d}_{1}, \cdots, \mathbf{d}_{m}\right)$ such that the product $r_{\mathbf{d}_{1}} \cdots r_{\mathbf{d}_{m}}$ in the above sum has the component $\left[N^{\prime}\right]$. Since $T$ is a homogeneous tube, it follows that $\left(\mathbf{d}_{1}, \cdots, \mathbf{d}_{m}\right) \in \Theta_{T}$ if and only if $r_{\mathbf{d}_{1}}, \cdots, r_{\mathbf{d}_{m}}$ all have indecomposable $T$ components. But $r_{\mathbf{d}_{i}}$ has an indecomposable $T$-component if and only if $\mathbf{d}_{i}=\mu_{i} \lambda \mathbf{n}$ with $\mu_{i}$ a positive integer. This proves $\Theta_{T}=\Theta_{T^{\prime}}$, which is denoted by $\Theta$ in the following.

Thus, we have

$$
[N]=\sum_{\left(\mathbf{d}_{1}, \cdots, \mathbf{d}_{m}\right) \in \Theta} c_{\mathbf{d}_{1}, \cdots, \mathbf{d}_{m}} r_{\mathbf{d}_{1}} \cdots r_{\mathbf{d}_{m}}+\sum_{\left(\mathbf{d}_{1}, \cdots, \mathbf{d}_{m}\right) \notin \Theta} c_{\mathbf{d}_{1}, \cdots, \mathbf{d}_{m}} r_{\mathbf{d}_{1}} \cdots r_{\mathbf{d}_{m}} .
$$

Let $X$ and $Y$ denote respectively the first and the second sum in the right hand of the above equality. Note that for every $\left(\mathbf{d}_{1}, \cdots, \mathbf{d}_{m}\right) \in \Theta, r_{\mathbf{d}_{i}}$ can be written as

$$
r_{\mathbf{d}_{i}}=\left[N_{i}\right]+\left[N_{i}^{\prime}\right]+X_{i}, \quad 1 \leq i \leq m,
$$

where $\left[N_{i}\right]$ and $\left[N_{i}^{\prime}\right]$ are respectively the indecomposable $T$ - and $T^{\prime}$-components of $r_{\mathbf{d}_{i}}$ (this means that $X_{i}$ has no indecomposable $T$ - or $T^{\prime}$-components). It follows that

$$
\begin{aligned}
X & =\sum_{\left(\mathbf{d}_{1}, \cdots, \mathbf{d}_{m}\right) \in \Theta} c_{\mathbf{d}_{1}, \cdots, \mathbf{d}_{m}} \prod_{1 \leq j \leq m}\left(\left[N_{i}\right]+\left[N_{i}^{\prime}\right]+X_{i}\right) \\
& =\sum_{\left(\mathbf{d}_{1}, \cdots, \mathbf{d}_{m}\right) \in \Theta} c_{\mathbf{d}_{1}, \cdots, \mathbf{d}_{m}}\left([N]+\left[N^{\prime}\right]+V\right) .
\end{aligned}
$$

Since $N$ and $N^{\prime}$ are regular serial, both the coefficients of $[N]$ and $\left[N^{\prime}\right]$ are 1 . Note that $V$ has no indecomposable $T$ - or $T^{\prime}$-components.

For every $\left(\mathbf{d}_{1}, \cdots, \mathbf{d}_{m}\right) \notin \Theta, r_{\mathbf{d}_{1}} \cdots r_{\mathbf{d}_{m}}$ has no indecomposable $T$ - or $T^{\prime}$-components. By comparing the coefficients of $[N]$ and $\left[N^{\prime}\right]$ in both sides of the equality

$$
[N]=\sum_{\left(\mathbf{d}_{1}, \cdots, \mathbf{d}_{m}\right) \in \Theta} c_{\mathbf{d}_{1}, \cdots, \mathbf{d}_{m}}\left([N]+\left[N^{\prime}\right]+V\right)+Y
$$


we then get the desired contradiction

$$
1=\sum_{\left(\mathbf{d}_{1}, \cdots, \mathbf{d}_{m}\right) \in \Theta} c_{\mathbf{d}_{1}, \cdots, \mathbf{d}_{m}}=0,
$$

which completes the proof.

5.3. Proof of Proposition 5.1. Let $E$ be the quasi-simple module in $T$. Note that $E$ is not a stone, and that $\operatorname{dim} M=m \operatorname{dim} E$ with $m$ a positive integer. If $m=1$, then $M=E$, and it follows from Lemma 5.2 that $[E] \notin \mathcal{C}(A)$. Let $m \geq 2$. Suppose that $[M] \in \mathcal{C}(A)$. Then by Theorem 0.4 we have $[M] \in \mathcal{T}$. Since $\delta_{E} \in \operatorname{Hom}_{\mathbb{Q}}(\mathcal{T}, \mathcal{T})$ by Theorem 3.5, it follows that $\delta_{E}^{m-1}([M])=\delta_{E} \cdots \delta_{E}([M]) \in \mathcal{T}$. Note that by induction and the definition of $\delta_{E}$ we have $\delta_{E}^{m-1}([M]) \neq 0$. On the other hand, let $\delta_{E}^{m-1}([M])=\sum c_{i}\left[X_{i}\right]$. Then for each $c_{i} \neq 0$ (note that the $c_{i}$ 's are positive numbers) we see that $X_{i} \in T$ and $\operatorname{dim} X_{i}=\operatorname{dim} M-(m-1) \operatorname{dim} E=$ $\operatorname{dim} E$. It follows that $X_{i}=E, \forall i, c_{i} \neq 0$, i.e. $\delta_{E}^{m-1}([M])=c[E]$ with $c \neq 0$, and then we get the desired contradiction $[E] \in \mathcal{T} \subseteq \mathcal{C}(A)$ by Lemma 5.2.

Remark. (i) We cannot apply Corollary 3.8 in the proof of Proposition 5.1, since $[E] \notin \mathcal{C}(A)$.

(ii) As we see from the proof, if Proposition 1.4 is also proved for non-simply-laced tame hereditary algebras, then Proposition 5.1 is proved for any tame hereditary algebra.

\section{REDUCTION STEPS}

In this section we shall prove the main result, Theorem 0.5 , which is a direct consequence of the following three theorems.

Theorem 6.1. Let $A$ be an arbitrary representation-infinite hereditary algebra over a finite field. Let $M=P \oplus R \oplus I$ be an A-module with $P, R$ and $I$ being preprojective, regular and preinjective, respectively. Then $[M] \in \mathcal{C}(A)$ if and only if $[R] \in \mathcal{C}(A)$.

Proof. If $[R] \in \mathcal{C}(A)$, then $[M]=[P] \cdot[R] \cdot[I] \in \mathcal{C}(A)$ since $[P],[I] \in \mathcal{C}(A)$ by [Z1. Theorem 1].

Conversely, let $[M] \in \mathcal{C}(A)$. Let $L$ be an $A$-module with $g_{L, I}^{M} \neq 0$. Then there exists a short exact sequence $0 \longrightarrow I \longrightarrow P \oplus R \oplus I \longrightarrow L \longrightarrow 0$. Since $\operatorname{Hom}_{A}(I, P \oplus R)=0$, it follows that $L=P \oplus R$ and $g_{P \oplus R, I}^{M}=1$, and hence by Theorem 3.7 we have

$$
\delta_{I}([M])=\sum_{[L]} g_{L, I}^{M} \frac{a_{L}}{a_{M}}[L]=g_{P \oplus R, I}^{M} \frac{a_{P \oplus R}}{a_{M}}[P \oplus R]=\frac{a_{P \oplus R}}{a_{M}}[P \oplus R] \in \mathcal{C}(A),
$$

which implies that $[P \oplus R] \in \mathcal{C}(A)$. Applying ${ }_{P} \delta$ to $[P \oplus R]$, by the similar argument we see that $[R] \in \mathcal{C}(A)$.

Theorem 6.2. Let $A$ be any tame hereditary algebra over a finite field. Let $R=$ $R_{1} \oplus \cdots \oplus R_{m}$ be a regular module with $R_{i} \in T_{i}$, where the $T_{i}, 1 \leq i \leq m$, are pairwise different tubes of $A$. Then $[R] \in \mathcal{C}(A)$ if and only if $\left[R_{i}\right] \in \mathcal{C}(A), \forall 1 \leq i \leq m$.

Proof. Since the $T_{i}, 1 \leq i \leq m$, are pairwise different tubes of $A$, it follows that $\operatorname{Hom}_{A}\left(R_{i}, R_{j}\right)=0=\operatorname{Ext}_{A}^{1}\left(R_{i}, R_{j}\right)$ for $i \neq j$, and hence $[R]=\left[R_{1}\right] \cdots\left[R_{m}\right]$, and from this the "if" part follows.

Conversely, let $[R] \in \mathcal{C}(A)$, and let $E_{1}$ be a quasi-simple in $T_{1}$ such that $\operatorname{Hom}_{A}\left(E_{1}, R_{1}\right) \neq 0$. Since the $T_{i}, 1 \leq i \leq m$, are pairwise different, it follows 
that $\operatorname{Hom}_{A}\left(E_{1}, R_{i}\right)=0, \forall i \neq 1$, and hence $\delta_{E_{1}}\left(\left[R_{i}\right]\right)=0, \forall i \neq 1$. Since $[R] \in \mathcal{T}$ by Theorem 0.4 , it follows that $\delta_{E_{1}}([R]) \in \mathcal{T}$ by Theorem 3.5 (notice that here we cannot apply Corollary 3.8 , since at this moment it is not clear whether or not $\left[E_{1}\right]$ is in $\mathcal{C}(A))$.

Let

$$
\delta_{E_{1}}\left(\left[R_{1}\right]\right)=\sum_{X} g_{X, E_{1}}^{R_{1}} \frac{a_{X}}{a_{R_{1}}}[X] .
$$

Then by Corollary 3.4 we have

$$
\begin{aligned}
\delta_{E_{1}}([R]) & =\delta_{E_{1}}\left(\left[R_{1}\right] \cdots\left[R_{m}\right]\right)=\delta_{E_{1}}\left(\left[R_{1}\right]\right)\left[R_{2}\right] \cdots\left[R_{m}\right] \\
& =\sum_{X} g_{X, E_{1}}^{R_{1}} \frac{a_{X}}{a_{R_{1}}}[X]\left[R_{2}\right] \cdots\left[R_{m}\right] \in \mathcal{T} .
\end{aligned}
$$

It should be pointed out here that at this moment it is not clear whether or not $\left[X \oplus R_{2} \oplus \cdots \oplus R_{m}\right]=[X] \cdot\left[R_{2}\right] \cdots\left[R_{m}\right]$ is in $\mathcal{T}$. However, since $X \in T_{1}$ and $\operatorname{dim} X<\operatorname{dim} R_{1}$, by repeating the above process finitely many times to the sum

$$
\sum_{X} g_{X, E_{1}}^{R_{1}} \frac{a_{X}}{a_{R_{1}}}[X]\left[R_{2}\right] \cdots\left[R_{m}\right]
$$

(but not to $[X] \cdot\left[R_{2}\right] \cdots\left[R_{m}\right]$ ) we see that $\left[R_{2}\right] \cdots\left[R_{m}\right] \in \mathcal{T}$. By induction on $m$ we know that every $\left[R_{i}\right] \in \mathcal{T}$.

Theorem 6.3. Let $A$ be the path algebra of a Euclidean quiver over a finite field $k$ with $|k| \geq 4$. Let $T$ be a tube of $A$ and $R$ a module with $R \in T$. Then $[R] \in \mathcal{C}(A)$ if and only if $T$ is non-homogeneous and $\mathbf{q} \cdot \operatorname{dim} M \not(1, \cdots, 1)$.

Proof. The sufficiency follows from Proposition 2.4, and the necessity from Proposition 5.1 and Proposition 4.1.

Remark 6.4. As we see from the proofs, throughout this paper, only in Propositions 1.1 and 1.4 have we really used the assumptions of $A$ being a path algebra and $|k| \neq 2,3$ - see Claim 2.8 and Lemma 5.2. That is, if we can be prove Claim 2.8 and Lemma 5.2 also for non-simply-laced tame hereditary algebras, then Theorem 0.5 is proved for any tame hereditary algebra over a finite field. We conjecture that Theorem 0.5 is true for any tame hereditary algebra over any finite field.

\section{Consequences}

Let $A$ be the path algebra of a Euclidean quiver over a finite field $k$ with $|k| \geq 4$. As a direct consequence of Theorem 0.5 we have

Corollary 7.1 ([ZZ] $)$. Let $M$ be an indecomposable A-module. Then $[M] \in \mathcal{C}(A)$ if and only if $M$ is a stone.

Let $T$ be a non-homogeneous tube of $A$. Recall that the composition algebra $\mathcal{C}(T)$ of $T$ is the subalgebra of $\mathcal{H}(A)$ generated by all quasi-simples in $T$. Note that $\mathcal{C}(T) \subseteq \mathcal{C}(A)$, and that the defining relations of $\mathcal{C}(T)$ have been completely determined in R7].

Corollary 7.2. Let $M$ be an A-module in $T$. Then $[M] \in \mathcal{C}(T)$ if and only if $[M] \in \mathcal{C}(A)$, if and only if $\mathbf{q} \cdot \operatorname{dim} M \not(1, \cdots, 1)$.

Proof. Assume that $[M] \in \mathcal{C}(A)$. Then by Theorem 0.5 we have q. $\operatorname{dim} M \not$ $(1, \cdots, 1)$, and hence by Proposition 2.4 we have $[M] \in \mathcal{C}(T)$. 
Remark. Corollary 7.2 positively answers a question asked by Yu. A. Drozd at the Conference of Representation Theory at Oberwolfach in 1995.

A pair $(U, V)$ of indecomposable $A$-modules is said to be separating provided $\operatorname{Ext}_{A}^{1}(V, U)=\operatorname{Ext}_{A}^{1}(U, U)=\operatorname{Ext}_{A}^{1}(V, V)=0$.

Corollary 7.3. Let $M$ be an A-module with $[M] \in \mathcal{C}(A)$, and let $T$ be a nonhomogeneous tube. Let $M_{1}$ and $M_{2}$ be two arbitrary indecomposable direct summands of $M$ with $M_{1}, M_{2} \in T$. Then at least one of the pairs $\left(M_{1}, M_{2}\right)$ and $\left(M_{2}, M_{1}\right)$ is separating.

Proof. Let $E_{1}, \cdots, E_{m}$ be the quasi-simples in $T$ with $\tau E_{i}=E_{i+1}, 1 \leq i \leq m-1$, and $\tau E_{m}=E_{1}$. Let $M_{1}=E_{1}\left(t_{1}\right)$ and $M_{2}=E_{j}\left(t_{2}\right)$ (recall that $E_{i}(t)$ denotes the indecomposable in $T$ with quasi-top $E_{i}$ and quasi-length $t$ ). Then by Theorem 0.5 we know that $1 \leq t_{1}, t_{2} \leq m-1$, and both $M_{1}$ and $M_{2}$ are stones. If $M_{1}, M_{2}$ have the same quasi-top $E_{1}$, then by the Auslander-Reiten formula and the structure of a non-homogeneous tube we see that $\operatorname{Ext}_{A}^{1}\left(M_{1}, M_{2}\right)=\operatorname{Ext}_{A}^{1}\left(M_{2}, M_{1}\right)=0$. So, it suffices to assume $2 \leq j \leq m$. Since $1 \leq t_{1}, t_{2} \leq m-1$, it is easy to see that q. $\operatorname{dim} M_{1}+$ q. $\operatorname{dim} M_{2} \not(1, \cdots, 1)$ if and only if

$$
j \leq \min \left\{t_{1}, m-t_{2}\right\} \text {, or } j=t_{1}+1, t_{2}<m-t_{1} \text {, or } j>t_{1}+1 ;
$$

and that $\operatorname{Ext}_{A}^{1}\left(M_{2}, M_{1}\right)=\operatorname{Ext}_{A}^{1}\left(E_{j}\left(t_{2}\right), E_{1}\left(t_{1}\right)\right)=\operatorname{Hom}_{A}\left(E_{1}\left(t_{1}\right), E_{j+1}\left(t_{2}\right)\right) \neq 0$ if and only if

$$
m-j+1 \leq t_{2} \leq t_{1}+m-j .
$$

Combining (7) and (8) we see that, if $\operatorname{Ext}_{A}^{1}\left(M_{2}, M_{1}\right) \neq 0$, then

$$
m+1 \leq t_{2}+j \leq m+t_{1} \text {, and } j>t_{1}+1 .
$$

This means $\operatorname{Ext}_{A}^{1}\left(M_{1}, M_{2}\right)=\operatorname{Ext}_{A}^{1}\left(E_{1}\left(t_{1}\right), E_{j}\left(t_{2}\right)\right)=\operatorname{Hom}_{A}\left(E_{j}\left(t_{2}\right), E_{2}\left(t_{1}\right)\right)=0$. (Otherwise, assume that $\operatorname{Hom}_{A}\left(E_{j}\left(t_{2}\right), E_{2}\left(t_{1}\right)\right) \neq 0$. Then by the same analysis we get $j-1 \leq t_{1} \leq t_{2}+j-2$, in particular, $j \leq t_{1}+1$, which contradicts (9).) This completes the proof.

\section{ONE APPLICATION}

The following lemma is well-known; see e.g. [Ker, 1.8].

Lemma 8.1. Let $Q$ be a wild quiver with $\left|Q_{0}\right| \geq 3$. Then there exists a sink or a source $i$ such that the full subquiver given by $Q_{0}-\{i\}$ is connected and not a Dynkin quiver.

Proof. This is based on the analysis on quivers. For example, one can start from all Dynkin quivers by adding a new sink or source in possible ways such that the new quiver $Q$ is wild; then one can delete a suitable vertex $i$ ( $i$ can even be chosen as a source or a sink) such that the corresponding full subquiver is not of Dynkin type.

Denote by $K(m)$ the path algebra of the quiver with two vertices 1 and 2 , and $m$ arrows from 1 to 2 .

Lemma 8.2. If $m \geq 2$, then $\mathcal{C}(K(m)) \neq \mathcal{H}(K(m))$.

Proof. One can easily verify that any indecomposable module with dimension vector $(1,1)$ does not lie in the composition algebra $\mathcal{C}(K(m))$. 
Lemma 8.3. Let $e$ be a primitive idempotent of $A$ and $B=A / A e A$. If $\mathcal{C}(A)=$ $\mathcal{H}(A)$, then $\mathcal{C}(B)=\mathcal{H}(B)$.

Proof. Let $S(i), 1 \leq i \leq n$, be the set of all simple $B$-modules. Then $S(i), 1 \leq$ $i \leq n$, and $S(e)$ are all the simple $A$-modules. Let $M \in B$-mod. Then as an $A$-module we have $[M] \in \mathcal{C}(A)$ by assumption, and hence $[M] \in \mathcal{C}(A)_{\mathbf{d}}$, where $\mathbf{d}=\left(d_{1}, \cdots, d_{n}, d_{e}\right)=\operatorname{dim} M$ with $d_{e}=0$. Thus $[M] \in \mathcal{C}(A)$ is a $\mathbb{Q}$-combination of all monomials $\left[S\left(i_{1}\right)\right] \cdots\left[S\left(i_{d}\right)\right]$ with $d=d_{1}+\cdots+d_{n}$, such that the number of occurences of $i$ in the sequence $i_{1}, \cdots, i_{d}$ is exactly $d_{i}$ for $1 \leq i \leq n$. Since $d_{e}=0$, and $B$-mod is an extension-closed full subcategory of $A$-mod, it follows that the monomial $\left[S\left(i_{1}\right)\right] \cdots\left[S\left(i_{d}\right)\right]$ has the same value in both $\mathcal{H}(A)$ and $\mathcal{H}(B)$. This proves $[M] \in \mathcal{C}(B)$, and hence $\mathcal{C}(B)=\mathcal{H}(B)$.

The sufficiency of the following theorem has been proved in [R5, Prop. 6].

Theorem 8.4. Let $A=k Q$ be the path algebra of a finite quiver $Q$ without oriented cycles, where $k$ is a finite field with $|k| \neq 2$. Then $\mathcal{H}(A)=\mathcal{C}(A)$ if and only if $Q$ is a Dynkin quiver, i.e. $A$ is of finite representation type.

Proof. We only need to prove the necessity. Let $\mathcal{H}(A)=\mathcal{C}(A)$. Use induction on $s=\left|Q_{0}\right|$.

If $s=2$, then by Lemma 8.2 we know that $Q$ has to be of type $A_{2}$.

Assume that $s \geq 3$. Then by Proposition 2.1 we know that $Q$ cannot be a Euclidean quiver. The following arguments show that $Q$ also cannot be a wild quiver: If $Q$ were wild, then by Lemma 8.1 there exists a path algebra $B$ such that $B=A / A e A$ is of infinite representation type. Then by induction we see $\mathcal{C}(B) \neq \mathcal{H}(B)$, which contradicts Lemma 8.3. This finishes the proof.

\section{ACKNOWLEDGEMEnts}

This work was partly done during the author's visit at Virginia Tech, USA, supported by a grant from the Chinese Education Ministry. I would like to thank Professor Edward L. Green for his hospitality, and the Department of Mathematics for the working facilities.

I also want to thank Professor Charles W. Curtis and the referee for valuable suggestions and remarks in revising the paper.

\section{REFERENCES}

[ARS] M. Auslander, I. Reiten and S. Smalø: Representation theory of Artin algebras, Cambridge Studies in Advanced Math. 36, Cambridge Univ. Press, 1995. MR 96c:16015

[C1] W. W. Crawley-Boevey, Lectures on representations of quivers; More lectures on representations of quivers, preprints, Oxford (1992).

[C2] W. W. Crawley-Boevey, Exceptional sequences of representations of quivers, Canad. Math. Soc. Conf. Proc. 14(1993) (V. Dlab and H. Lenzing, eds.), 117-124. MR 94c:16017, MR 94j:16002

[CX] X. Chen and J. Xiao: Exceptional sequences in Hall algebras and quantum groups, Compositio Math. 117(2)(1999), 165-191. CMP 99:14

[DR] V. Dlab and C. M. Ringel: Indecomposable representations of graphs and algebras, Mem. Amer. Math. Soc. 173 (1976). MR 56:5657

[Ga] P. Gabriel: Unzerlegbare Darstellungen I, Manuscripta Math. 6 (1972), 71-103. MR 48:11212

[G1] J. A. Green: Hall algebras, hereditary algebras and quantum groups, Inventiones Math. 120(1995), 361-377. MR 96c:16016 
[G2] J. A. Green: Quantum groups, Hall algebras and quantized shuffles, In: Finite reductive groups, related structures and representations (M. Cabanes, ed.), Birkhäuser, Boston, (1997), 273-290. MR 98h:16061

[GL] W. Geigle and H. Lenzing: Perpendicular categories with applications to representations and sheaves, J. Algebra 144(1991), 273-343. MR 93b:16011

[GP] J. Y. Guo and L. G. Peng: Universal PBW-basis of Hall - Ringel algebras and Hall polynomials, J. Algebra 198(1997), 339-351. MR 99b:16033

[H] D. Happel: Triangulated categories in the representation theory of finite dimensional algebras, London Math. Soc. Lect. Note Ser. 119, Cambridge Univ. Press, 1988. MR 89e:16035

[HHKU] D. Happel, S. Hartlieb, O. Kerner and L.Unger: On perpendicular categories of stones over quiver algebras, Comment. Math. Helvetici 71(1996) 463-474. MR 98b:16013

[J] N. Jacobson: Basic algebra I, second edition, W.H.Freeman and Company, New York 1985. MR 86d:00001

[K1] V. G. Kac: Infinite dimensional Lie algebras, Progress in Math. Vol. 44, Birkhäuser, Boston. Basel. Stuttgart 1983. MR 86h:17015

[K2] V. G. Kac: Infinite root systems, representations of graphs and invariant theory, Inventiones Math. 56(1980), 57-92. MR 82j:16050

[K3] V. G. Kac: Infinite root systems, representations of graphs and invariant theory II, J. Algebra 78(1982), 141-162. MR 85b:17003

[Kap] M. Kapranov: Eisenstein series and quantum affine algebras, J. Math. Sci. 84(1997), 1311-1360. MR 98i:11033

[Ker] O. Kerner: Representations of wild quivers, Canad. Math. Soc. Conf. Proc. Vol. 19 (1996) (R. Bautista, R. Martinez-Villa and J. A. de la Peña, eds.), 65-107; Amer. Math. Soc. Providence RI 1996. MR 97e:16028

[L1] G. Lusztig: Canonical basis arising from quantized enveloping algebras, J. Amer. Math. Soc. 3(1990), 447-498. MR 90m:17023

[L2] G. Lusztig: Quivers, perverse sheaves and quantized enveloping algebras, J. Amer. Math. Soc. 4(1991), 365-421. MR 91m:17018

[L3] G. Lusztig: Introduction to quantum groups, Birkhäuser, Boston, 1993. MR 94m:17016

[Mac] I. G. Macdonald: Symmetric functions and Hall polynomials, Second Edition. Oxford University Press, Oxford. Basel. New York, 1995. MR 96h:05207

[M] R. V. Moody: Euclidean Lie algebras, Canadian J. Math. 21(1969), 1432-1454. MR 41:287

[R1] C. M. Ringel: Tame algebras and integral quadratic forms, Lecture Notes in Math. 1099, Springer-Verlag, Berlin, New York, 1984. MR 87f:16027

[R2] C. M. Ringel: Hall algebras, In: Topics in Algebra, Banach Center Publ. 26(1990), 433-447. MR 93f:16027

[R3] C. M. Ringel: Hall polynomials for the representation-finite hereditary algebras, Adv. Math. 84(1990), 137-198. MR 92e:16010

[R4] C. M. Ringel: Hall algebras and quantum groups, Inventiones Math. 101(1990), 583-592. MR 91i:16024

[R5] C. M. Ringel: From representations of quivers via Hall and Loewy algebras to quantum groups, Contemporary Math. 131 (Part 2) (1992), 381-401. MR 93h:17065

[R6] C. M. Ringel: Lie algebras arising in representation theory, In: Representation of Algebras and Related Topics (H.Tachikawa and S.Brenner, eds.), London Math. Lect. Note Ser. 168, Cambridge Univ. Press (1992), 284-291. MR 94d:16020

[R7] C. M. Ringel: The composition algebra of a cyclic quiver, Proc. London Math. Soc.(3) 66 (1993), 507-537. MR 94g:16013

[R8] C. M. Ringel: The Hall algebra approach to quantum groups, Aportaciones Mathemáticas, Comunicaciones 15 (1995), 85-114. [MR 96m:17034]

[R9] C. M. Ringel: Hall algebras revisited, Israel Mathematica Conference Proceedings. Vol.7 (1993), 171-176. MR 94k:16021

[R10] C. M. Ringel: PBW-bases of quantum groups, J. Reine Angew. Math. 470(1996), 51-88. MR 97d:17009

[R11] C. M. Ringel: Green's Theorem on Hall algebras, Canad. Math. Soc. Conf. Proc. Vol. 19 (1996) (R. Bautista, R. Martinez-Villa and J. A. de la Peña, eds.), 185-245. Amer. Math. Soc. Providence RI 1996. MR 97h:16014 
[R12] C. M. Ringel: The braid group action on the set of exceptional sequences of a hereditary artin algebra, Contemporary Math. 171(1994), 339-352. MR 95m:16006

[Rm] Chr. Riedtmann: Lie algebras generated by indecomposables, J. Algebra 170(1994), 526546. MR 96e: 16013

[S] A. Schofield: Semi-invariants of quivers, J. London Math. Soc.43(1991), 385-395. MR 92g:16019

[Z1] P. Zhang: Triangular decomposition of the composition algebra of the Kronecker algebra, J. Algebra 184(1996), 159-174. MR 97j:16018

[Z2] P. Zhang: Indecomposables in the composition algebra of the Kronecker algebra, Comm. in Algebra 27 (1999), 4733-4639. CMP 99:17

[Z3] P. Zhang: Composition algebras of affine types, J. Algebra 206(1998), 505-540. CMP 98:16

[ZZ] P. Zhang and S. H. Zhang: Indecomposables as elements in affine composition algebras, J. Algebra 210(1998), 614-629. CMP 99:05

Department of Mathematics, University of Science and Technology of China, Hefei 230026, People's Republic of China

E-mail address: pzhang@ustc.edu.cn 